\title{
Mutual editing of alternative splicing between breast cancer cells and macrophages
}

\author{
WANBAO DING $^{1 *}$, DONGDONG LI $^{3 *}$, PEIXIAN ZHANG $^{1}$, LAN SHI $^{1}$, HUI DAI $^{1}$,

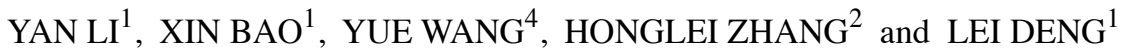 \\ ${ }^{1}$ Key Laboratory of Tumor Immunological Prevention and Treatment, Department of Oncology, \\ Yan'an Affiliated Hospital of Kunming Medical University, Kunming, Yunnan 650118; \\ ${ }^{2}$ State Key Laboratory of Genetic Resources and Evolution, Kunming Institute of Zoology, Chinese Academy of Sciences, \\ Kunming, Yunnan 650223; ${ }^{3}$ Anhui Medical University, Hefei, Anhui 230022; ${ }^{4}$ Department of Breast Cancer, \\ Third Affiliated Hospital, Kunming Medical University, Kunming, Yunnan 650118, P.R. China
}

Received December 12, 2018; Accepted June 6, 2019

DOI: 10.3892/or.2019.7200

\begin{abstract}
Breast cancer is a highly heterogeneous disease and numerous secreted factors may differentially contribute to a macrophage phenotype whose extensive infiltration is generally regarded as indicative of an unfavorable outcome. How different breast tumor cells and macrophage cells interplay or influence each other on the alternative splicing (AS) level have not been characterized. Here, we exploited one previous study, which investigated the interplay between macrophages and estrogen receptor-positive $\left(\mathrm{ER}^{+}\right)$breast cancer and triple-negative breast cancer (TNBC) at the transcriptional level, to investigate the tumor-macrophage crosstalk at the AS level. In the present study, it was demonstrated that biological processes such as DNA damage and DNA repair were significantly affected both in $\mathrm{ER}^{+}$breast cancer and TNBC by co-culturing with macrophages, whereas biological pathways altered in macrophages co-cultured with tumor cells depended on the breast cancer type. Specifically, biological processes altered in macrophages co-cultured with $\mathrm{ER}^{+}$breast cancer were enriched in RNA processing and translation-related pathways whereas biological processes altered in macrophages
\end{abstract}

Correspondence to: Dr Lei Deng, Key Laboratory of Tumor Immunological Prevention and Treatment, Department of Oncology, Yan'an Affiliated Hospital of Kunming Medical University, 245 East of Renmin Road, Kunming, Yunnan 650118, P.R. China

E-mail: 1033498884@we.com

Mr. Honglei Zhang, State Key Laboratory of Genetic Resources and Evolution, Kunming Institute of Zoology, Chinese Academy of Sciences, 32 Jiaochang Donglu, Kunming, Yunnan 650223, P.R. China E-mail: hlzhang2014@163.com

*Contributed equally

Key words: breast cancer, macrophage, alternative splicing, DNA repair, splicing factor co-cultured with TNBC were mainly enriched in protein transport pathways. We also analyzed the sequence features of skip exons among different conditions. In addition, putative splicing factors which were responsible for the altered AS profile in each condition were identified. The findings of the present study revealed significant tumor-macrophage crosstalk at the AS level which may facilitate the development of new therapeutic strategies for cancer.

\section{Introduction}

Tumor-associated macrophages (TAMs), one main immune cell population found in most solid tumors, contribute to tumor development, progression, drug resistance and suppression of antitumor immune cells (1-4). There are two main TAM subtypes: M1 macrophages, which encourage inflammation, and M2 macrophage, which encourage tissue repair (5). TAMs are mainly of the M2 phenotype (6). TAMs are recruited to the tumor as a response to cancer-associated inflammation $(7,8)$ and acquire M2 properties in response to cytokines such as tumor growth factor (TGF)- $\beta$, interleukin (IL)-10 and macrophage colony-stimulating factor (M-CSF). An increased amount of M2 type TAMs is associated with a worse prognosis in several cancer types including breast cancer $(9,10)$. However, recent single-cell sequencing results have shown that both M1 and M2-associated genes are frequently expressed in the same cells and are positively correlated with one another along the same activation trajectory. These results have challenged the model of macrophage polarization wherein M1 and M2 activation states are two discrete states (11). As macrophages affect all therapeutic modalities, further investigation of the interplay between macrophages and tumor cells is urgently needed.

The interplay between tumor cells and macrophage have been revealed. For example, macrophages can release inflammatory compounds such as tumor necrosis factor (TNF)- $\alpha$ which activates nuclear factor $(\mathrm{NF})-\kappa \mathrm{B}$ and $\mathrm{NF}-\kappa \mathrm{B}$ regulates tumor cell apoptosis, proliferation and inflammation. Moreover, macrophages serve as a source for many pro-angiogenic factors including vascular endothelial growth 
factor (VEGF), TNF- $\alpha$, M-CSF/CSF1 and IL-1 and IL-6 further contributing to tumor growth and metastasis (12). In turn, tumors produce factors, such as $\mathrm{M}-\mathrm{CSF} / \mathrm{CSF} 1$, monocyte chemoattractant protein-1 (MCP-1)/CCL2 and angiotensin II, triggering the amplification and mobilization of macrophages in tumors (13-15).

Alternative splicing (AS) is a post-transcriptional regulation process during gene expression that results in a single gene coding for multiple proteins. AS has been proved to play important roles in macrophage differentiation and regulation of most tumor hallmarks (16). For example, Liu et al found that splicing factor $M B N L 1$ is the major regulator during the differentiation from monocytes to macrophages (17). Human M-CSF heterogeneity is partially derived from alternative mRNA splicing (18). However, how tumor cells and macrophages interplay at the alternative splicing level and its potential application in cancer therapy remain unknown. Understanding the tumor-macrophage crosstalk at the AS level would reveal the molecular mechanisms underlying tumor progression, drug resistance and suppression of immune cells and further shade light on the identification of new cancer therapeutic strategies.

Generally, TAMs appear to have an unfavorable role in breast cancer $(19,20)$. Moreover, breast cancer is considered to be a highly heterogeneous disease, and many secreted factors may differentially contribute to the macrophage phenotype. Therefore, the aim of the present study was to understand macrophage 'education' under the influence of two breast cancer types, estrogen receptor-positive $\left(\mathrm{ER}^{+}\right)$breast cancer and triple-negative breast cancer (TNBC) at the AS level in a simplified setting. Through calculation of the number of common and specific altered AS events, the present study aimed to ascertain: i) Whether $\mathrm{ER}^{+}$breast cancer and TNBC cells exert similar influences on co-cultured macrophage cells; ii) whether $\mathrm{ER}^{+}$breast cancer and TNBC cells are affected to a similar degree at the AS level in the presence of macrophages; iii) which specific biological pathways are disturbed at the AS level in macrophage cells when cocultured with $\mathrm{ER}^{+}$breast cancer and TNBC cells; iv) which specific biological pathways are disturbed at the AS level in $\mathrm{ER}^{+}$breast cancer and TNBC cells at the presence of macrophage cells.

In the present study, we reanalyzed a previously published dataset (GSE75130) and our results showed that differing from transcriptional analysis, DNA repair and DNA damage processes were enriched in both $\mathrm{ER}^{+}$breast cancer and TNBC after co-culturing with macrophages, which indicated the conserved functional regulation of macrophages on tumor cells in the tumor microenvironment. Meanwhile, macrophages were differentially regulated from the pathway views by co-culturing with $\mathrm{ER}^{+}$breast cancer and TNBC cells. Sequence features of skipped exons from different conditions were also characterized in our analysis.

\section{Materials and methods}

GEO dataset GSE75130 was reanalyzed (21) which was originally used to characterize the differences in macrophage activation under the influence of either $\mathrm{ER}^{+}$breast cancer or TNBC cells. For detailed treatment procedures please refer to a previous study (21).
Profiling of gene expression. Paired-end reads were mapped to the human genome primary assembly (GRCh37) (22), and the Ensembl human gene annotation for GRCh37 genebuild was used to improve the accuracy of the mapping with STAR software (STAR_2.4.2a) (23). FeatureCounts (version 1.4.6-p5) (24) was used to assign sequence reads to genes. Mitochondrial genes, ribosomal genes, and genes possessing less than five raw reads in half the samples were removed. Normalized gene expression profiles were obtained with the edgeR package 1.6 (25).

Alternative splicing analysis. For AS analysis, MISO software (version 0.5.4) (26) was used to analyze RNA-Seq data and estimate the percentage of splicing isoforms. Firstly, we utilized MISO to achieve the AS profiles for each treated sample and 5 types of AS profiles including alternative $3^{\prime} / 5^{\prime}$ splice site (A3SS, A5SS), skipped exons (SE), mutually exclusive exons (MXE) and retained introns (RI) were obtained. AS profiles were represented by percentage of splice-in (PSI/ $\Psi)$ values. Secondly, we identified the delta PSI ( $\triangle$ PSI) by comparing the cocultured sample with individually cultured sample. Finally, we determine the significantly different AS events by Bayes factor $>6$ and $|\Delta \mathrm{PSI}|>0.2$.

Principal component analysis (PCA). PCA was performed as previously described (27). A total of 20,169 genes were accounted for in PCA of the whole transcriptome. Expression was normalized with the reads per million mapped reads (RPM) method. The prcomp package from $\mathrm{R}$ was used to perform PCA and the default parameters were used (28). The ggplot 2 package from $\mathrm{R}$ was used to draw the scatter plot (29). A total of 36,377 AS events were utilized in the PCA of the $\mathrm{ER}^{+}$breast cancer and TNBC transcriptome analysis. A total of 35,476 AS events were utilized in the PCA of the macrophage transcriptome analysis.

Functional enrichment analysis. Gene Ontology (GO) term enrichment analysis was conducted with webserver DAVID 6.8 (30). $\mathrm{P}<0.05$ was considered as statistically significant. Background gene list was set to 16,133 esemble genes which possess alternative splicing isoforms.

Protein-protein interaction analysis. Web server STRING (31) was utilized to explore the protein-protein interactions. Default parameters were applied.

NCBI protein domain analysis. Protein domains of full-length CHEK2 (accession no. CAG30304.1) was analyzed with NCBI webserver (https://www.ncbi.nlm.nih.gov) (32). Two significant protein domains were identified. The first domain is STKc_Chk2 (accession: cd14084) and the second is FHA (accession: cd00060).

Evolutionary conservation. Placental Mammal PhastCons scores were used to represent evolutionary conservation. For average conservation of exons, bigWigAverageOverBed (33) was used to calculate the mean conservation across each exon. The ggplot2 package from $\mathrm{R}$ was used to draw the box plot (29). 
3' and 5'splicing strength. To evaluate splice site strength, 3' of the exon-intron boundary ( $20 \mathrm{nt}$ into intron and $+3 \mathrm{nt}$ into exon) and $5^{\prime}$ of the exon-intron boundary ( $3 \mathrm{nt}$ into exon and $+6 \mathrm{nt}$ into intron), together with the transcript sequences for these regions were obtained by bedtools (34). MaxEntScan (35) was used to calculate the strength of the splice sites for the AS exons.

Repetitive element enrichment. To identify repetitive elements in AS exons, Repeat Masker track was downloaded from UCSC Genome Browser and intersected with AS exons by bedtools intersect (34). Repeats were grouped into families defined by the Pfam database of repetitive DNA elements. Phylostratum scores were used to describe gene age, as previously reported (36).

Association between 222 splicing factors and altered AS events. Overlap of gene-associated events and conditional specific events (macrophages vs. macrophages co-cultured with T47D cells; macrophages vs. macrophages co-cultured with MDA-MB-231 cells; T47D cells vs. T47D cells co-cultured with macrophages; and MDA-MB-231 cells vs. MDA-MB-231 cells co-cultured with macrophages) were used to assess how much of the conditional specific events could be explained by each splicing factor. First, conditional specific ES events were isolated by comparing splicing events from treatment samples against those from the control samples. The significant splicing events were determined based on Bayes factor $>6$ and $\mid \Delta$ PSII $>0.2$. Second, gene-associated ES events were determined by correlation analyses using gene expression levels (RPKM) and PSI values across all samples. Correlation coefficients (R-value) and corresponding P-values were calculated with the Pearson method, and gene-associated events were determined by $\mathrm{R}>0.2$ and $\mathrm{P}<0.0001$. Common events were considered as events that occurred (overlapped) in both gene-associated and conditional specific events. Finally, top 5 splicing factors which explain the most conditional specific events were isolated.

Statistical analyses. Significantly different AS events were determined by Bayes factor $>6$ and $|\triangle \mathrm{PSI}|>0.2$ during comparison of AS events between cocultured state and single cultured state. Fisher exact test was applied to test significance of GO term enrichment and $\mathrm{P}<0.05$ was considered as statistically significant. $\mathrm{R}>0.2$ and $\mathrm{P}<0.0001$ were applied to identify significantly associated AS events to a specific splicing factor across 14 samples in this study. Wilcoxon signed-rank test was applied to test significance in Fig. $6 \mathrm{~A}-\mathrm{D}$ and $\mathrm{P}<0.05$ was set to determine significance. Fisher's exact test was used to test significant enrichment of each elements and P-values were- $\log 10$ transformed to plot the heatmap in Fig. 6E.

\section{Results}

Confirmation of data reliability. To investigate the interplay between breast cancer cells and macrophages at the alternative splicing level, we downloaded one dataset from a previous study (21). In this previous study, freshly isolated human peripheral monocytes were cultured with two breast cancer cell lines (T47D, ER ${ }^{+}$and MDA-MB-231, TNBC) in an in vitro Transwell co-culture assay. Then, at day five, the whole transcriptome of macrophage cells and breast tumor cells were sequenced. Detailed sample information was previously described (22). We firstly evaluated the data reliability by investigating the genetic variance among samples using gene expression profiles based on the assumption that samples from the same cell type and treatment should have smaller genetic distances. PCA was performed with 20,169 genes and the results showed that samples were grouped together according to the types and treatments (Fig. 1A). Then unsupervised clustering based on the same transcriptomes confirmed the dataset reliability (Fig. 1B).

DNA damage and DNA repair pathways are altered in both MDA-MB-231 and T47D cell lines by co-culturing with macrophages. Next, we aimed to ascertain how macrophages educate different breast cancer cells at the AS level. To obtain the AS profile for each sample, we performed the Mixture of Isoforms (MISO) pipeline (26) according to its recommended procedure (https://miso.readthedocs.io/en/fastmiso/). Five types of AS events including skip exon (SE), alternative $3^{\prime} / 5^{\prime}$ splice sites (A3SS, A5SS), mutually exclusive exons (MXE), and retained introns (RI) were analyzed. A total of 36,377 splicing events with values at least in 7 samples were identified. Then, PCA and unsupervised clustering were used to characterize the genetic distances among samples. The PCA result showed that both T47D and MDA-MB-231 individually cultured samples were obviously separated from their macrophage co-cultured states (Fig. 2A). Unsupervised clustering result also supported that co-cultured breast cancer cells were different from the individually cultured states (Fig. 2B). These results indicated that $\mathrm{AS}$ profiles of both $\mathrm{TNBC}$ and $\mathrm{ER}^{+}$breast cancer were affected by co-culturing with macrophages.

Then, it was further determined which AS events were affected in each breast caner type and whether the altered AS events from two breast cancer types were largely similar or different. We isolated those altered splicing events by comparing the co-cultured states with individually cultured states from T47D and MDA-MB-231 cell lines, respectively. Statistically different splicing events with delta percentage of splice-in $(\Delta \mathrm{PSI})>0.2$ and bayes factor $>6$ were isolated. A total of 101 and 108 different splicing events were identified from T47D and MDA-MB-231 cell lines, respectively (Tables I and II) and there were 10 common splicing events in both T47D and MDA-MB-231 altered splicing events. All of these results indicated that $\mathrm{ER}^{+}$breast cancer cells and TNBC cells were differently 'educated' at the AS level by co-culturing with the macrophages (Fig. 2C).

To further investigate the biological processes affected by those altered AS events, Gene Ontology (GO) functional enrichment analysis was performed. GO terms such as DNA repair, nucleotide-excision repair, DNA damage recognition, response to reactive oxygen species, intrinsic apoptotic signaling pathway in response to DNA damage were significantly enriched in the MDA-MB-231-altered AS events (Fig. 2D). Biological processes such as DNA damage response, signal transduction by p53 class mediator resulting in cell cycle arrest, stress-activated MAPK cascade, signal transduction in response to DNA damage were significantly enriched in T47D-altered alternative splicing events (Fig. 2E). Taken together, although the altered AS events from MDA-MB-231 and T47D cells were different, DNA repair and 
A

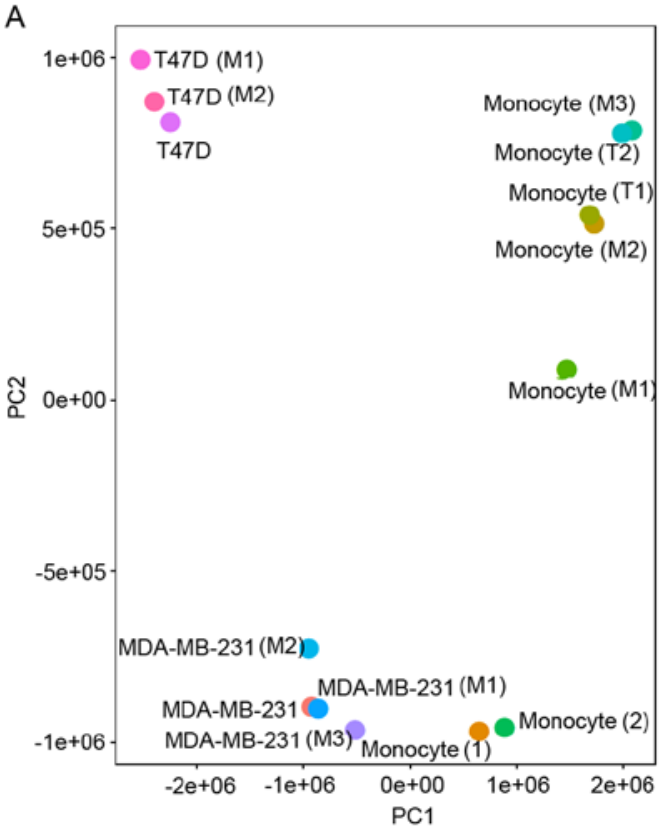

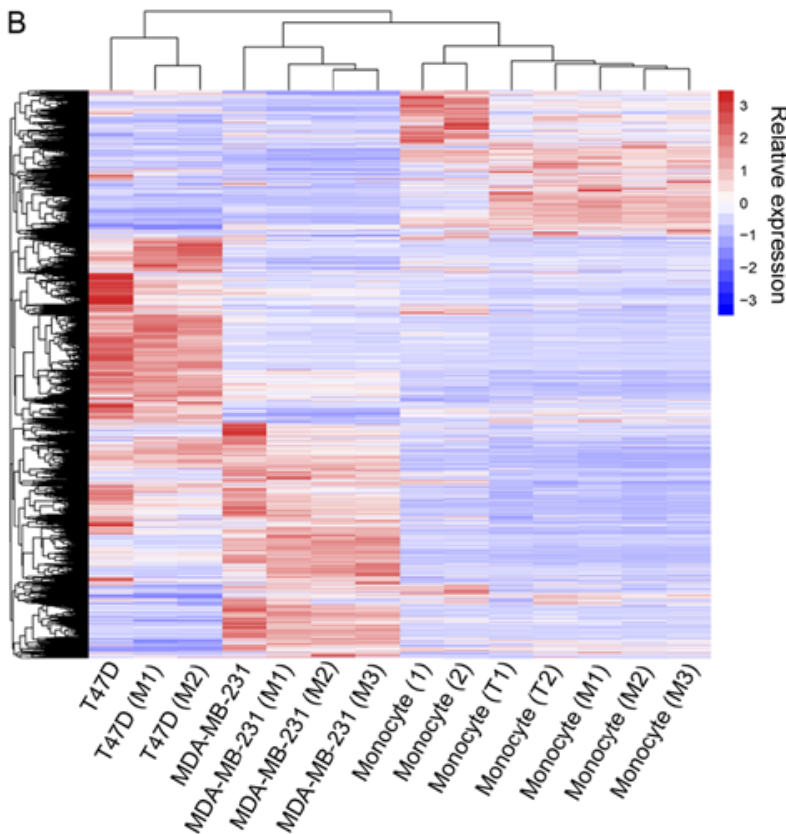

Figure 1. Data quality verification. (A) Principal component analysis (PCA) of whole expression profiles of the individually cultured macrophages [Monocyte (1) and Monocyte (2)] and of T47D [Monocyte (T1), Monocyte (T2)] or MDA-MB-231 [Monocyte (M1), Monocyte (M2), Monocyte (M3)] co-cultured macrophages; individually cultured T47D cells and co-cultured T47D cells [T47D (M1), T47D (M2)]; individually cultured MDA-MB-231 cells and cocultured MDA-MB-231 cells [MDA-MB-231 (M1), (MDA-MB-231 (M2), (MDA-MB-231 (M3)] which showed genetic distances among these samples. (B) Unsupervised clustering analysis of genetic distances of samples from A.

DNA damage-related biological processes were significantly enriched in both breast cancer cell lines of differing types in the presence of macrophages.

Then, we investigated how the DNA repair and DNA damage-related AS events influence the function of corresponding genes. Fig. 3A and $\mathrm{B}$ shows the DNA repair and DNA damage-related AS events identified from MDA-MB-231 and T47D samples, respectively. Sashimi plots showed the AS events 'chr22:29095826:29095925:-@chr22:29092889:29092975:-@ chr22:29091698:29091861:-' from gene Chek2 in T47D individual cultured sample (SRR2922618) and co-cultured sample (SRR2922619) (Fig. 3C). CHEK2 is a cell cycle checkpoint regulator and putative tumor suppressor (37) which contains a forkhead-associated protein interaction domain essential for activation in response to DNA damage and is rapidly phosphorylated in response to replication blocks and DNA damage. CHEK2 has been shown to stabilize the tumor-suppressor protein p53 $(38,39)$, leading to cell cycle arrest in $\mathrm{G} 1$ and it also interacts with and phosphorylates BRCA1, allowing BRCA1 to restore survival after DNA damage (40). According to our domain analysis, we found that CHEK2 contains two crutial functional domains (Fig. 3D): The first one is STKc_Chk2, in which STKs can catalyze the transfer of a $\gamma$-phosphoryl group from ATP to serine/threonine residues on protein substrates, and the second one is FHA domain which is a putative nuclear signaling domain. The skipped exon of Chek 2 encodes 28 aa which locates in the STKc_Chk2 domain (Fig. 3D). Thus the inclusion or exclusion of the skippd exon changes the STKc_Chk2 domain of Chek2, which may play an important role in the functional regulation of CHEK2. Protein-protein interaction network analysis showed that these DNA repair proteins have extensive interactions and CHEK2 may serve as a hub gene in the network (Fig. 3E).
Altered AS events in macrophages by co-culturing with MDA-MB-231 and T47D cells, respectively. As the differentiation of TAMs is largely regulated by mediators secreted by tumor cells, it was ascertained how different breast cancer cells 'educate' macrophages and regulate the differentiation process at the AS level. Similarly, a total of 35,476 splicing events with values in at least 7 samples were identified. Then, PCA and unsupervised clustering were used to characterize the genetic distances among samples. From the PCA result, it was revealed that macrophages individually cultured were separated from their co-cultured states whereas the co-cultured states of macrophage from T47D and MDA-MB-231 cells were not able to be separated (Fig. 4A), which is also supported by the unsupervised clustering result (Fig. 4B). Then, we isolated those altered splicing events by comparing the co-cultured samples with individually cultured samples. A total of 115 and 118 significant splicing events were identified in macrophages co-cultured with MDA-MB-231 and T47D cells, respectively (Tables III and IV). There were 14 common splicing events in the macrophages co-cultured with T47D and MDA-MB-231 cells $(\mathrm{P}>0.2)$ which indicated that $\mathrm{ER}^{+}$breast cancer cells and TNBC cells have different influences on macrophages at the AS level (Fig. 4C).

Similarly, GO functional enrichment analysis was applied to investigate the biological processes affected by those altered AS events. GO terms such as signal transduction, cell-matrix adhesion, protein transport, brain development were significantly enriched in the macrophages co-cultured with MDA-MB-231 cells (Fig. 4D). Terms such as RNA export from the nucleus, regulation of angiogenesis, positive regulation of transcription, DNA-templated, mRNA export from the nucleus, negative regulation of apoptotic process were significantly enriched in the macrophages co-cultured with the T47D 

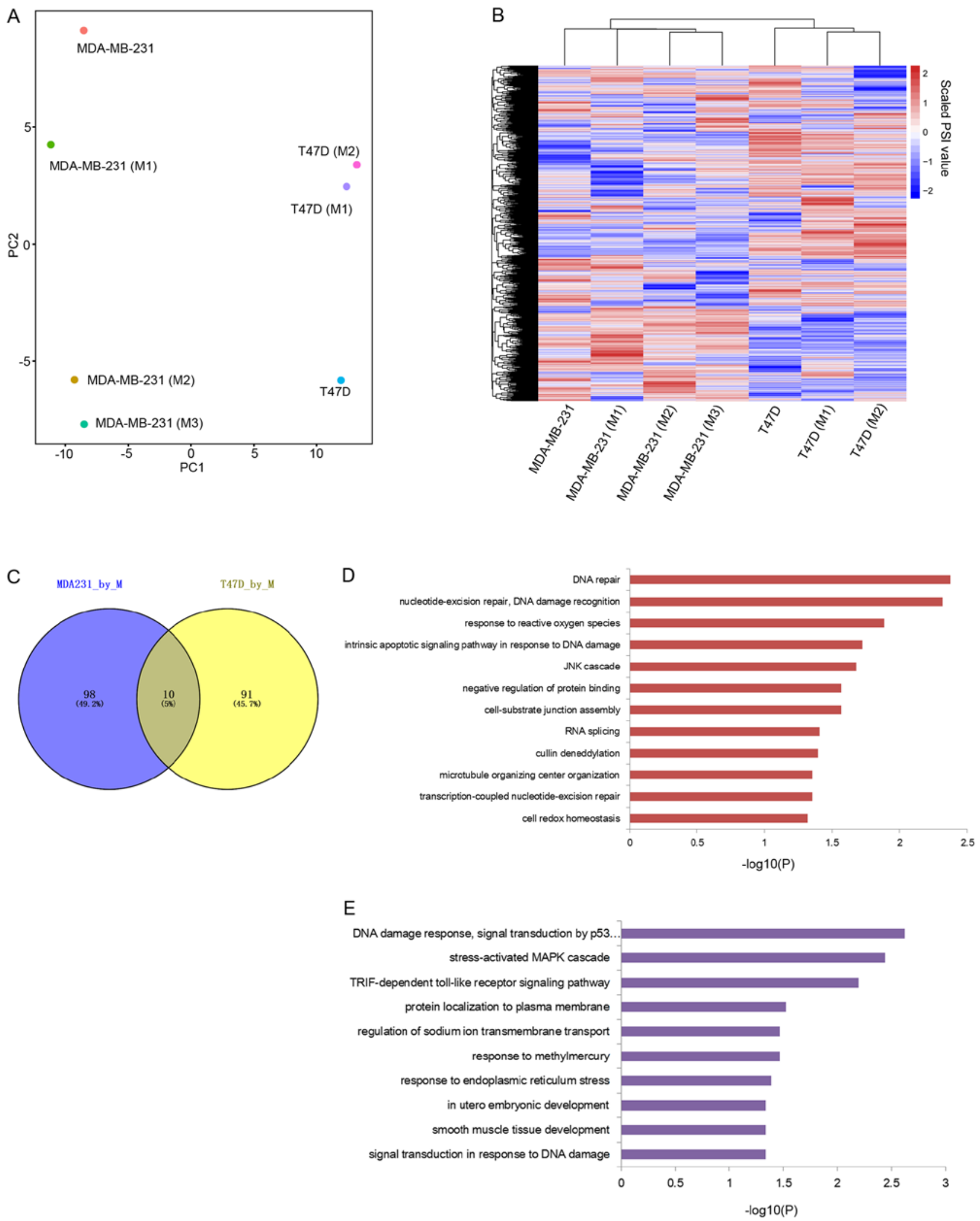

Figure 2. AS alteration in TNBC (MDA-MB-231) and $\mathrm{ER}^{+}$(T47D) breast cancer cells following co-culturing with macrophages. (A) Principal component analysis (PCA) of whole AS profiles of individually cultured T47D cells and co-cultured T47D cells; individually cultured MDA-MB-231 cells and cocultured MDA-MB-231 cells. (B) Clustering analysis of genetic distance of samples from (A). (C) Overlap of AS events between events altered in MDA-MB-231 cells cocultured with macrophages and events altered in T47D cells cocultured with macrophages. (D and E) Gene Ontology analysis of genes containing altered AS events in MDA-MB-231 cells co-cultured with macrophages (D) and genes containing altered AS events in T47D cells co-cultured with macrophage (E). AS, alternative splicing; TNBC, triple-negative breast cancer; ER+, estrogen receptor-positive.

cells (Fig. 4E). Briefly, protein transport-related processes were altered by co-culturing macrophages with the MDA-MB-231 cells and RNA processing biological processes were affected by co-culturing macrophages with the T47D cells. 


\begin{tabular}{|c|c|c|c|}
\hline Events & DNA repair related genes & Average delta PSI & Type \\
\hline chr3:9796388:9796569:+@echr3:9798451:9798500:+@chr3:9807493:9808353:+ & OGG1 & 0.43 & SE \\
\hline chr2:74685527:74685798:+@chr2:74685959:74686046:+@chr2:74686565:74686872:+ & INO80B & 0.54 & SE \\
\hline chr17:5329291-5329619:+@chr17:5331391-5331531: + & RPANN & 0.28 & R \\
\hline chr14:20923737-20923932:+@chr14:20924073-20924260:+ & APEX1 & 0.31 & $\mathrm{Ro}$ \\
\hline chr3:51976361:51976724:+@chr3:51977365|51977370:51977554:+ & PARP3 3 & -0.34 & A3SS \\
\hline chr16:74700684:74700779:-@chr16:74698522:74698643:-@chr16:74694830:74695349:-@chr16:74685818:74686020:- & RFWD3 & 0.23 & MXE \\
\hline chr2:232646417:232646593:+@chr2:232653265:232653442:+@chr2:232658973:232659061:+@ehr2:232660816:232661018:+ & COPS7B & -0.35 & MXE \\
\hline chr17:80009763:80009840:+@chr17:80010135:80010335:+@chr17:80011150:80011242:+ & GPS1 & 0.63 & SE \\
\hline chr12:125398665:125398551|125398587:-@ehr12:125398042:125398320:- & UBC & -0.25 & A5SS \\
\hline chr19:49464067-49464171:+@ehr19:49464789-49465055:+ & BAX & 0.23 & $\mathrm{R}$ \\
\hline chr14:24845500:24845760|24846084:+@chr14:24846844:24848810:+ & NFATC4 & 0.34 & A5SS \\
\hline chr6:160103670-160103506:-@chr6:160100330-160100096:- & SOD2 & 0.34 & $\mathrm{Ro}$ \\
\hline
\end{tabular}

\begin{tabular}{|c|c|c|c|}
\hline Events & DNA repair related genes & Average delta PSI & Type \\
\hline chr16:58663632:58663790:-@chr16:58658557:58658698:-@chr16:58657186:58657313:-@chr16:58633140:58633415:- & CNOT1 & -0.415 & MXE \\
\hline chr22:29095826:29095925:-@chr22:29092889:29092975:-@chr22:29091698:29091861:- & CHEK2 & -0.26 & SE \\
\hline chr1:155160639:155160707:-@ehr1:155160198:155160334:-@chr1:155159701:155159850:- & MUC1 & -0.355 & SE \\
\hline
\end{tabular}

C

chr22:29095826:29095925:-@chr22:29092889:29092975:-@chr22:29091698:29091861:-

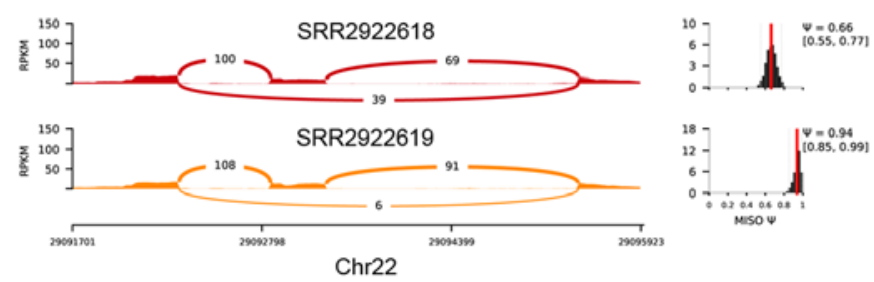

D

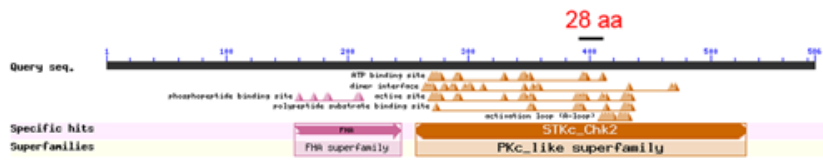

Figure 3. DNA repair-related AS events altered in MDA-MB-231 and T47D cells by co-culturing with macrophages. (A and B) DNA repair-related AS events altered in MDA-MB-231 cells cocultured with macrophages (A) and T47D cells co-cultured with macrophages (B). (C) Example of AS events from CHEK2 gene in T47D cells individually cultured (SRR2922618) and co-cultured with macrophages (SRR2922619). Numbers indicate read counts supporting the splicing junctions. $\Psi$ indicates PSI (percentage of splice-in). (D) Two functional domains identified in CHEK2: FHA domain and STKc_Chk2 domain. 28 aa was the amino acids encoded by the skip exon which is marked in black. (E) Protein-protein interaction (PPI) among DNA repair-related proteins. Colored edges represent active interaction sources (https://string-db.org/). AS, alternative splicing. AS, alternative splicing.

The RNA processing and protein transport-related AS events derived from the macrophages co-cultured with the MDA-MB-231 (Fig. 5A) and T47D (Fig. 5B) cells were further analyzed. Vacuolar protein sorting-associated protein 33B (VPS33B) is a Sec1 familiy protein involved in vesicle transport which mediates protein sorting and plays an important role in segregation of intracellular molecules into distinct organelles $(41,42)$. As known, the induced inflammatory responses by microbial ligands is influenced by internalization of Toll-like receptors (TLRs), thus, endosomal maturation in clearing receptors terminates inflammatory responses. Akbar et al found that Vps33B proteins play critical roles in the maturation of phagosomes and endosomes in Drosophila and mammals and therefore affect the process of maturation of macrophages (43). Sashimi plot showed the AS event 'chr15:91565384:91565833:-@ chr15:91561035:91561115:-@chr15:91557614:91557663:-' of gene VPS33B. The exon was largely excluded from the macrophages co-cultured with MDA-MB-231 cells (Fig. 5C), which may influence the protein function and further the maturation process of macrophages. The protein encoded by SRSF 6 belongs to the splicing factor SR and may play a role in the determination of alternative splicing. Boisson et al demonstrated that SRSF6 regulate the AS of the IKBKG gene, which accounts for NF- $\mathrm{\kappa B}$ activation and further immune response activation (44). Here, we showed that SRSF6 itself is regulated by AS. Specifically, the event 'chr20:42087001:42087149:+@ chr20:42087793:42088060:+@chr20:42088411:42088535:+’ of SRSF6 was significantly included after coculturing macrophages with T47D cells (Fig. 5C). PPI analysis showed that protein transport-related proteins CPNE1, AP1G2, AFTPH, STX2, VPS33B and STXBP2 were interacted intimately (Fig. 5D). In the interaction network of RNA processing proteins, SRSF6 has extensive interactions with another 6 proteins and may serve as the hub protein in the network (Fig. 5D). These results further indicate that AS events containing genes including $V p s 33 B$ and SRSF6 may play important roles in the maturation processes of macrophages.

Sequence feature characterization of the altered skip exons. To investigate whether the altered AS events in different treatments 

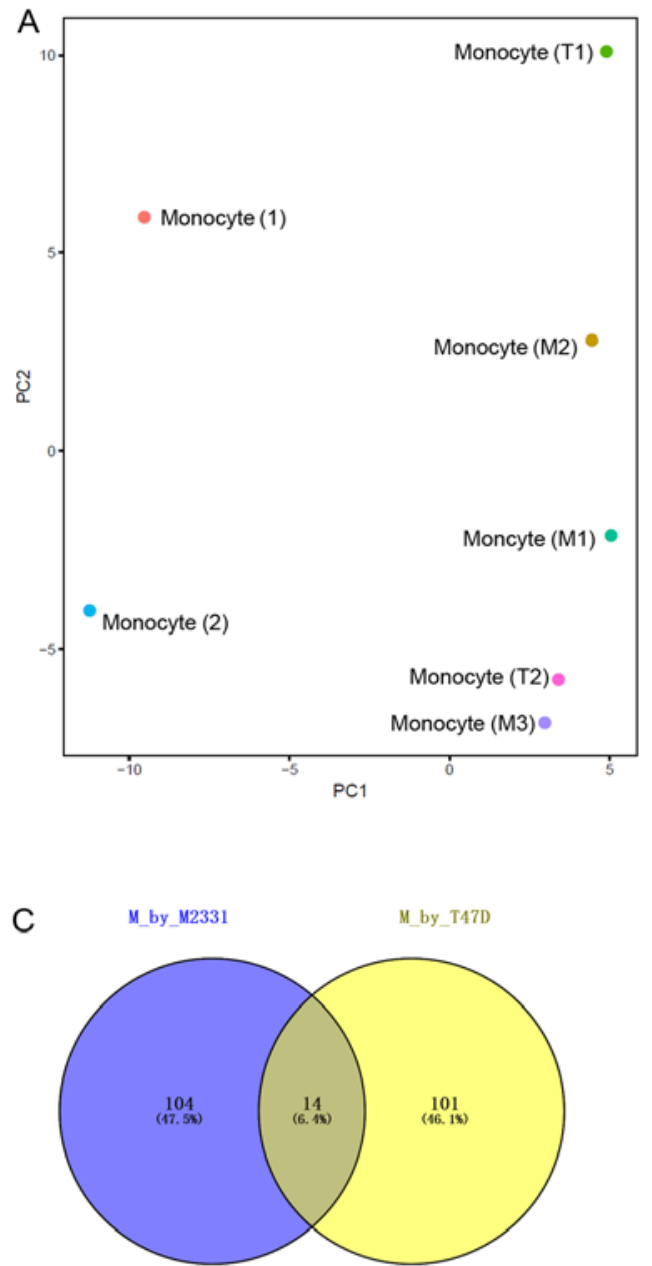

B

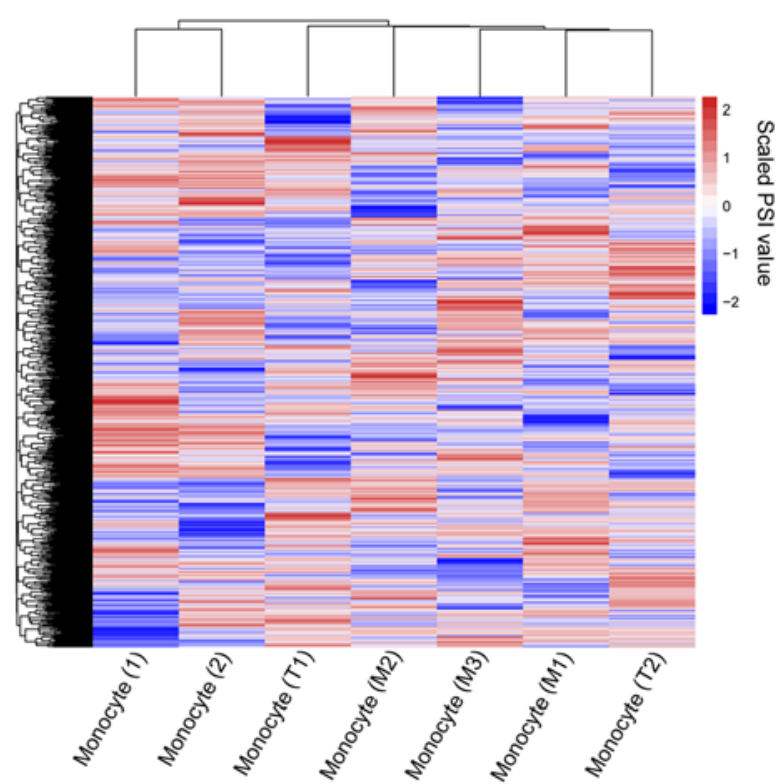

D

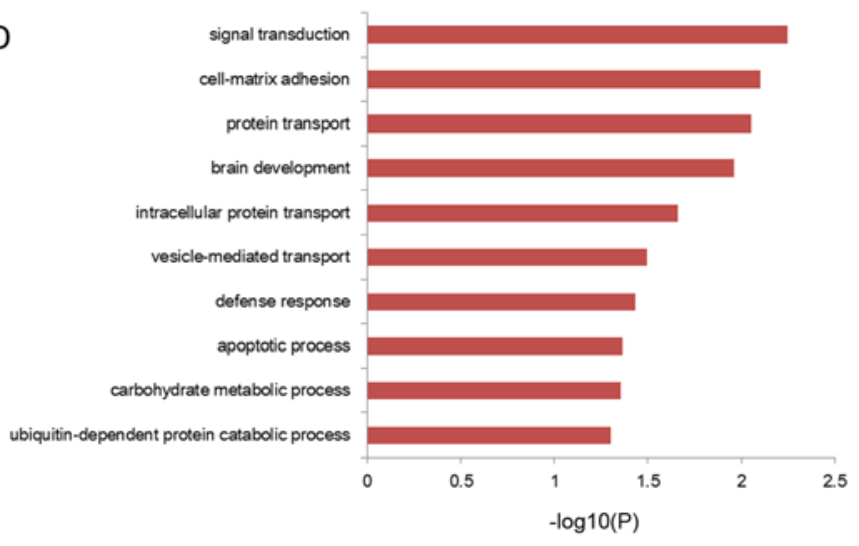

$\mathrm{E}$

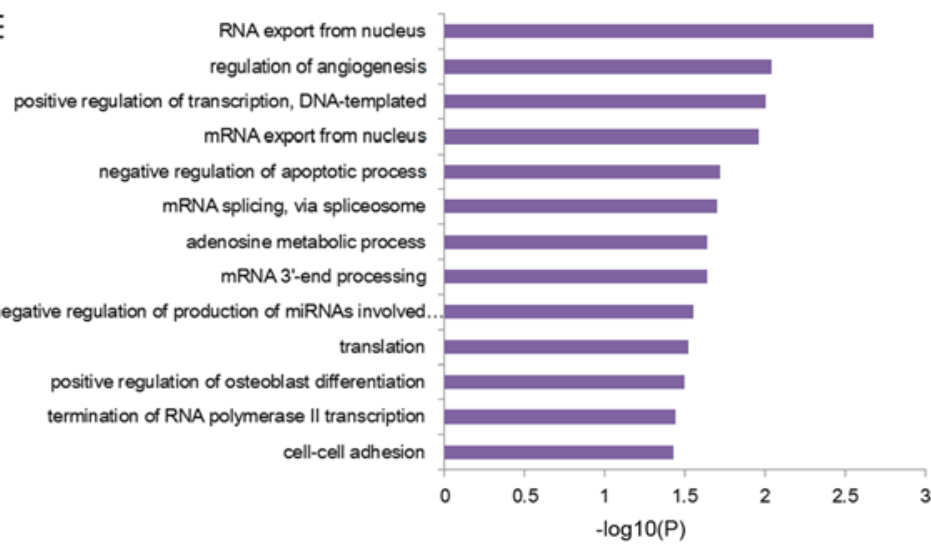

Figure 4. AS alteration in macrophages co-cultured with TNBC (MDA-MB-231) and ER ${ }^{+}$(T47D) breast cancer cells. (A) Principal component analysis (PCA) of whole alternative splicing profiles of individually cultured macrophages and of T47D or MDA-MB-231 cells co-cultured with macrophages. (B) Unsupervised clustering analysis of genetic distance of samples from (A). (C) Overlap of AS events between events altered in macrophages co-cultured with MDA-MB-231 cells and events altered in macrophages co-cultured with T47D cells. (D and E) Gene Ontology analysis of genes containing altered AS events in macrophages cocultured with MDA-MB-231 cells (D) and genes containing altered AS events in macrophages cocultured with T47D cells. (E) AS, alternative splicing; TNBC, triple-negative breast cancer; $\mathrm{ER}^{+}$, estrogen receptor-positive.

had distinct properties, we first measured the degree of evolutionary conservation of skip exon sequences across placental mammals. Unexpectedly, all the altered skip exons in different treatments showed a higher degree of sequence conservation equivalent compared to that of constitutive exons (Fig. 6A). Altered skip exons in the macrophages co-cultured with T47D cells showed highest evolutionary conservation, whereas macrophages co-cultured with MDA-MB-231 cells showed 
A

\begin{tabular}{lccc}
\hline \multicolumn{1}{c}{ Events } & \multicolumn{2}{c}{ Protein transport Average delta } \\
& related & PSI & Type \\
\hline chr5:39388901:39388954:-@chr5:39388407:39388469:-@chr5:39382720:39383373:-@chr5:39381556:39381718:- & DAB2 & -0.245 & MXE \\
chr11:18557953:18558016:-@chr11:18555877:18556000:-@chr11:18552950:18554034:- & UEVLD & -0.38 & SE \\
chr15:91565384:91565833:-@chr15:91561035:91561115:-@chr15:91557614:91557663:- & VPS33B & -0.305 & SE \\
chr2:64800080:64800202:+@chr2:64806620:64806680:+@chr2:64812556:64812679:+ & AFTPH & 0.465 & SE \\
chr10:46252460:46252587:+@chr10:46254763:46254849:+@chr10:46258836:46258937:+ & FAM21C & -0.285 & SE \\
chr11:3832480:3832654:+@chr11:3838662:3838765:+@chr11:3845113:3845365:+ & PGAP2 & 0.3 & SE \\
chr19:7707869:7707934:+@chr19:7708051:7708131:+@chr19:7709500:7709638:+ & STXBP2 & -0.285 & SE \\
chr14:24031497:24031624:-@chr14:24031171:24031275:-@chr14:24030721:24030844:- & AP1G2 & -0.37 & SE \\
chr20:34214725:34214489|34214657:-@chr20:34213953:34214303:- & CPNE1 & -0.25 & A5SS \\
chr12:131283070:131283180:-@chr12:131280540:131280665:-@chr12:131274145:131276522:- & STX2 & 0.335 & SE \\
chr1:174780970:174781098:+@chr1:174846530:174846743:+@chr1:174926594:174926686:+ & RABGAP1L & -0.44 & SE \\
chr12:72287002:72287104:+@chr12:72288105:72288155:+@chr12:72288466:72288663:+ & TBC1D15 & 0.215 & SE
\end{tabular}

B

\begin{tabular}{lccc}
\hline \multicolumn{1}{c}{ Events } & RNA & Average \\
& exportsplicing/translation & delta & Type \\
& related & PSI & \\
\hline chr1:153610771:153610924:+@chr1:153614719:153614905:+@chr1:153617540:153618782:+ & CHTOP & -0.31 & SE \\
chr19:50432583:50432988:-@chr19:50431072|50431105:50430951:- & NUP62 & -0.245 & A3SS \\
chr20:42087001:42087149:+@chr20:42087793:42088060:+@chr20:42088411:42088535:+ & SRSF6 & 0.32 & SE \\
\hline
\end{tabular}

C

chr15:91565384:91565833:-@chr15:91561035:91561115:-@chr15:91557614:91557663:-

$\left.\begin{array}{c}150 \\ 3 \\ 3 \\ 200 \\ 50\end{array}\right]$

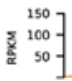<smiles>C1=C[C@H]2C[C@@H]1C2</smiles>

ง.s56:
SRR2922608

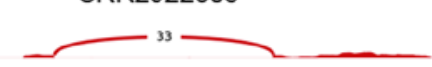

SRR2922609

Chr15

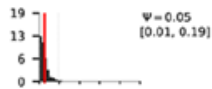

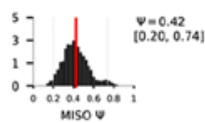

chr20:42087001:42087149:+@chr20:42087793:42088060:+@chr20:42088411:42088535:+
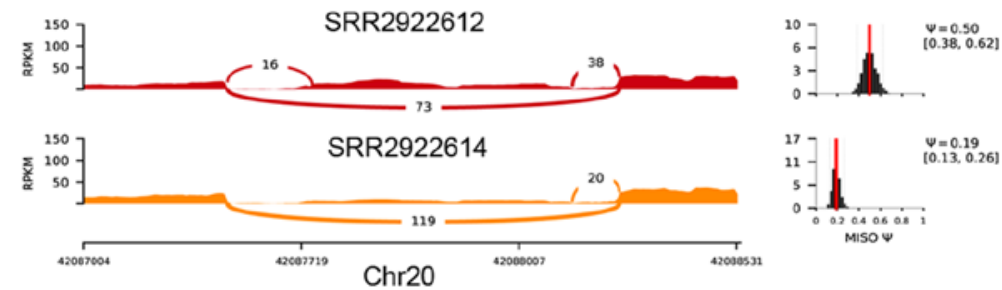

D
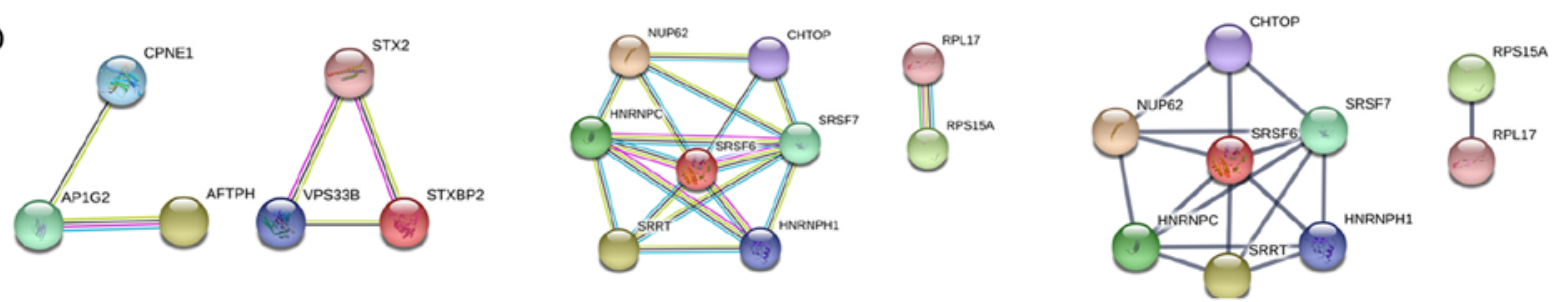

Figure 5. Protein transport and RNA export/splicing/translation-related AS events altered in macrophages co-cultured with MDA-MB-231 and T47D cells, respectively. (A) Protein transport-srelated AS events altered in macrophage co-cultured with MDA-MB-231 cells. (B) RNA export/splicing/translation-related AS in macrophages cocultured with T47D cells. (C) Example of AS event from the VPS33B gene in macrophages individually sampled (SRR2922608) and co-cultured with MDA-MB-231 cells (SRR2922609). AS event from the SRSF6 gene in macrophages individually sampled (SRR2922612) and co-cultured with T47D cells (SRR2922614). Numbers indicate read counts supporting the splicing junctions. $\Psi$ indicates PSI (percentage of splice-in). (D) Protein-protein interaction (PPI) among proteins from A and B. Colored edges represent active interaction sources (https://string-db.org/). AS, alternative splicing.

the lowest. There were no significant differences in skip exon length among the different treatments (Wilcoxon signed-rank test, P>0.05; Fig. 6B). Splicing site strength analysis showed there were no significant differences in splicing strength 

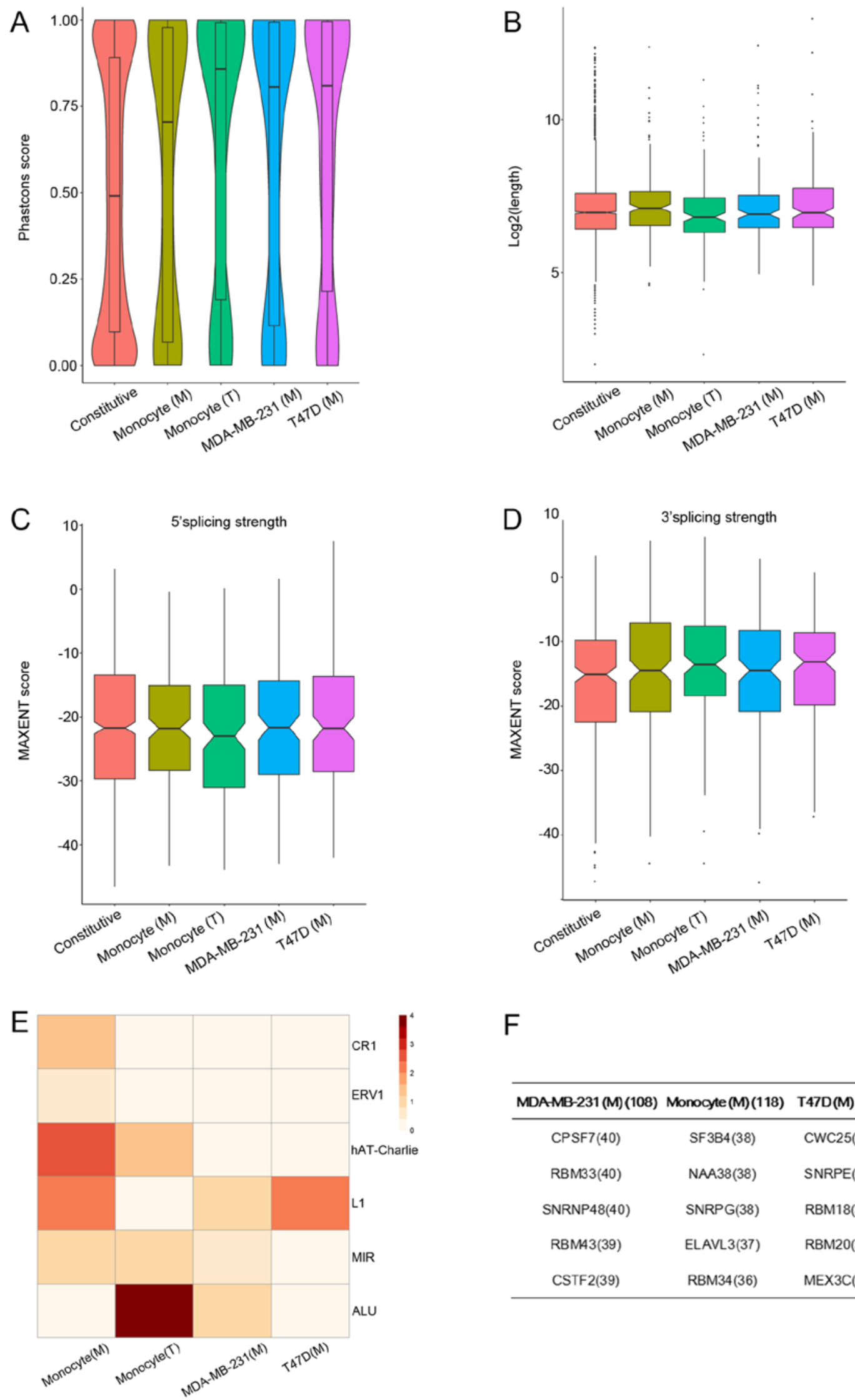

$\mathrm{F}$

MDAMB-231(M) (108) Monocyte(M)(118) T47D(M)(101) Monocyte(T)(115

\begin{tabular}{cccc}
\hline CPSF7(40) & SF3B4(38) & CWC25(33) & PRPF3(46) \\
RBM33(40) & NAA38(38) & SNRPE(33) & SF3B2(40) \\
SNRNP48(40) & SNRPG(38) & RBM18(32) & SNRNP48(40) \\
RBM43(39) & ELAVL3(37) & RBM20(31) & CELF1(40) \\
CSTF2(39) & RBM34(36) & MEX3C(31) & RBM6(39) \\
\hline
\end{tabular}

Figure 6. Sequence features of AS events. (A) Violin plot showing the Phastcon score distribution of constitutive exons, exons from altered ES type events of macrophages co-cultured with MDA-MB-231 cells [Monocyte (M)], exons from altered ES type events of macrophages co-cultured with T47D [Monocyte (T)], exons from altered ES type events of MDA-MB-231 cells co-cultured with macrophages [MDA-MB-231 (M)] and exons from altered ES type AS events of T47D cells cocultured with macrophages [T47D (M)]. (B) Boxplot showing the $\log 2$ transformed skip exon length across samples. (C) Boxplot showing the 5'splicing strength across samples. (D) Boxplot showing the 3' splicing strength across samples. (E) DNA element enrichment in Monocyte (M), Monocyte (T), MDA-MB-231 (M) and T47D (M). Constitutive skip exons were set as background. P-value derived hypergeometric distribution test and -log10(P) value was color scaled. (F) Top 5 splicing factors which were putatively responsible for the altered AS profiles in each condition. AS, alternative splicing. 


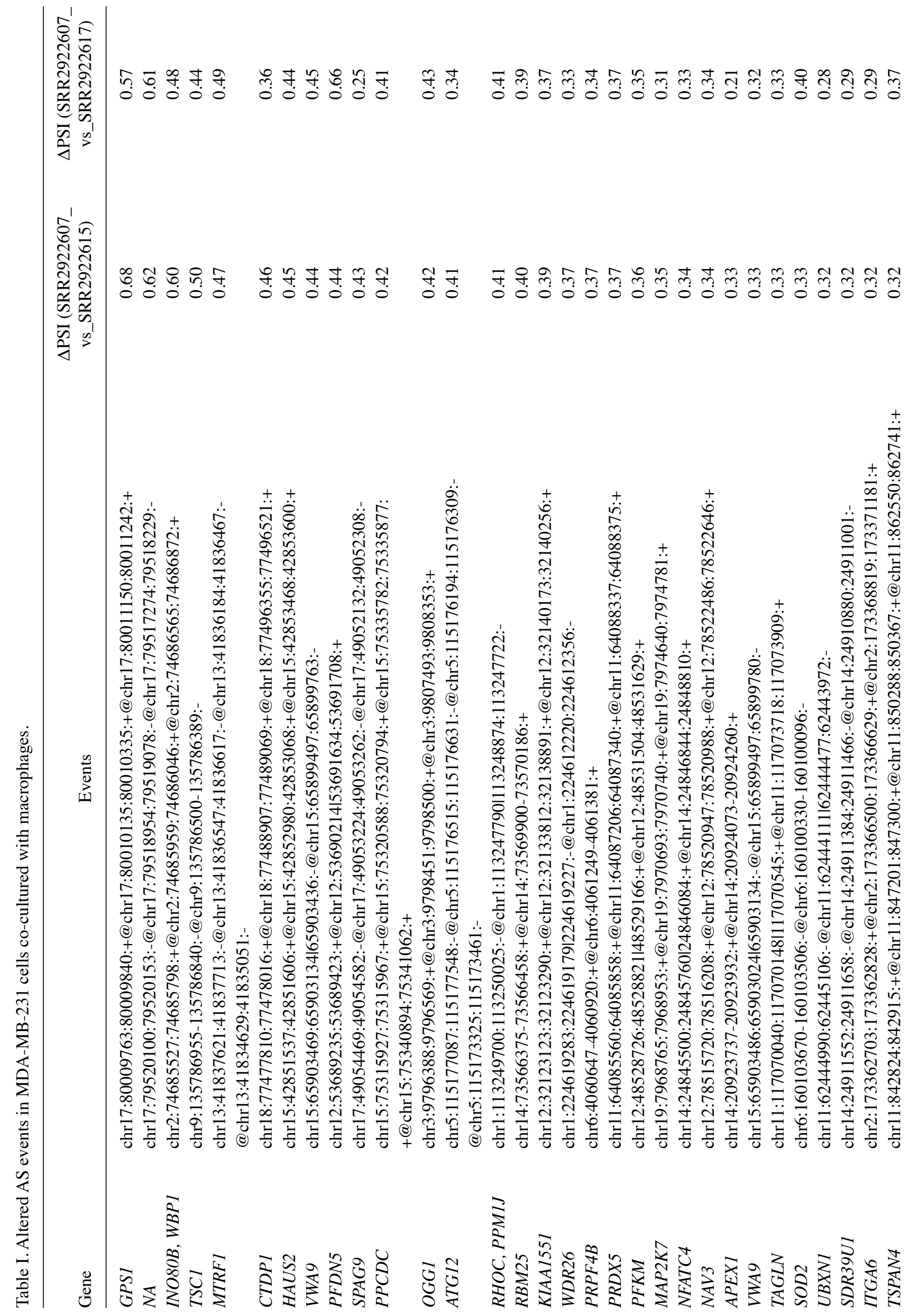




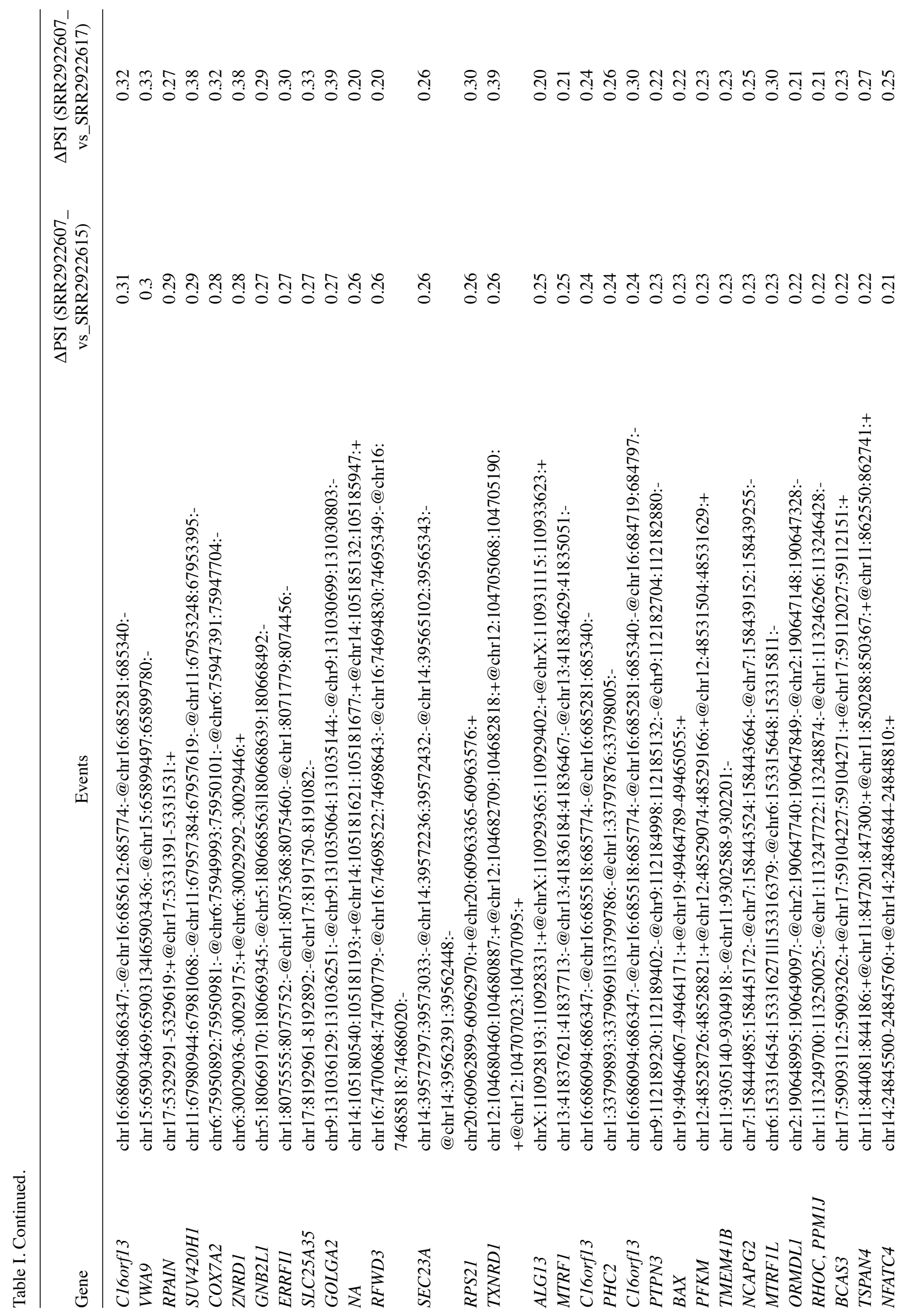




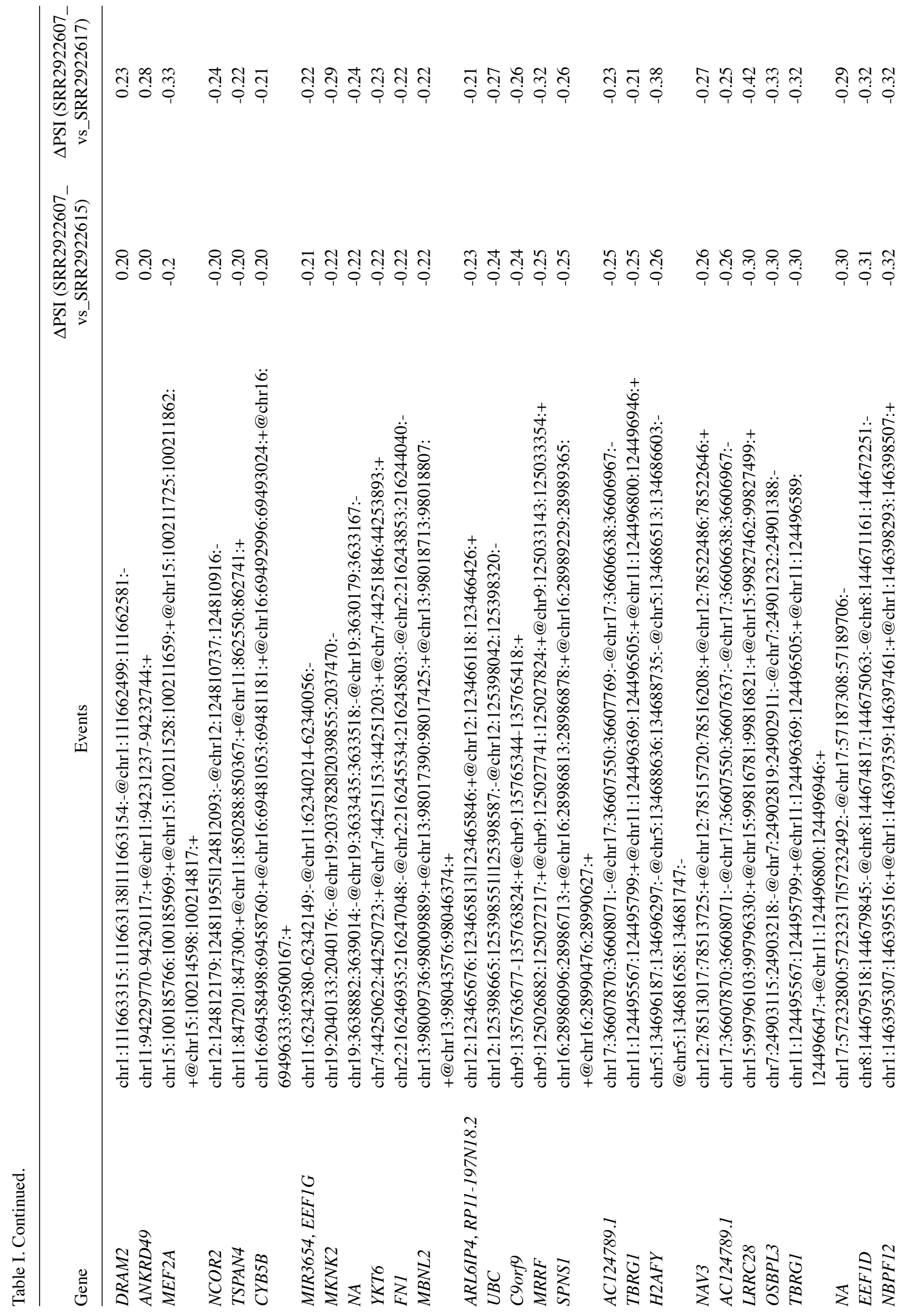




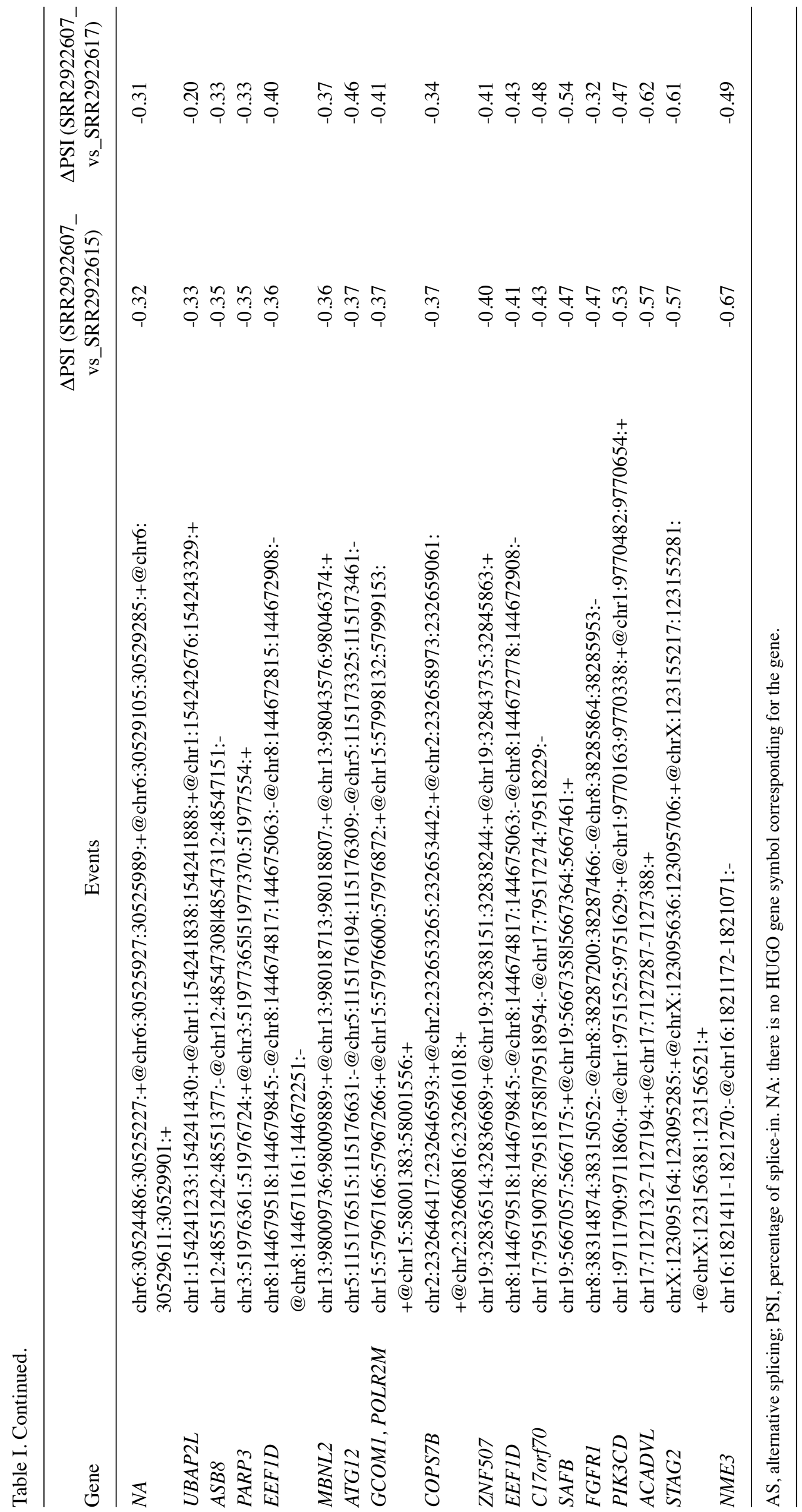




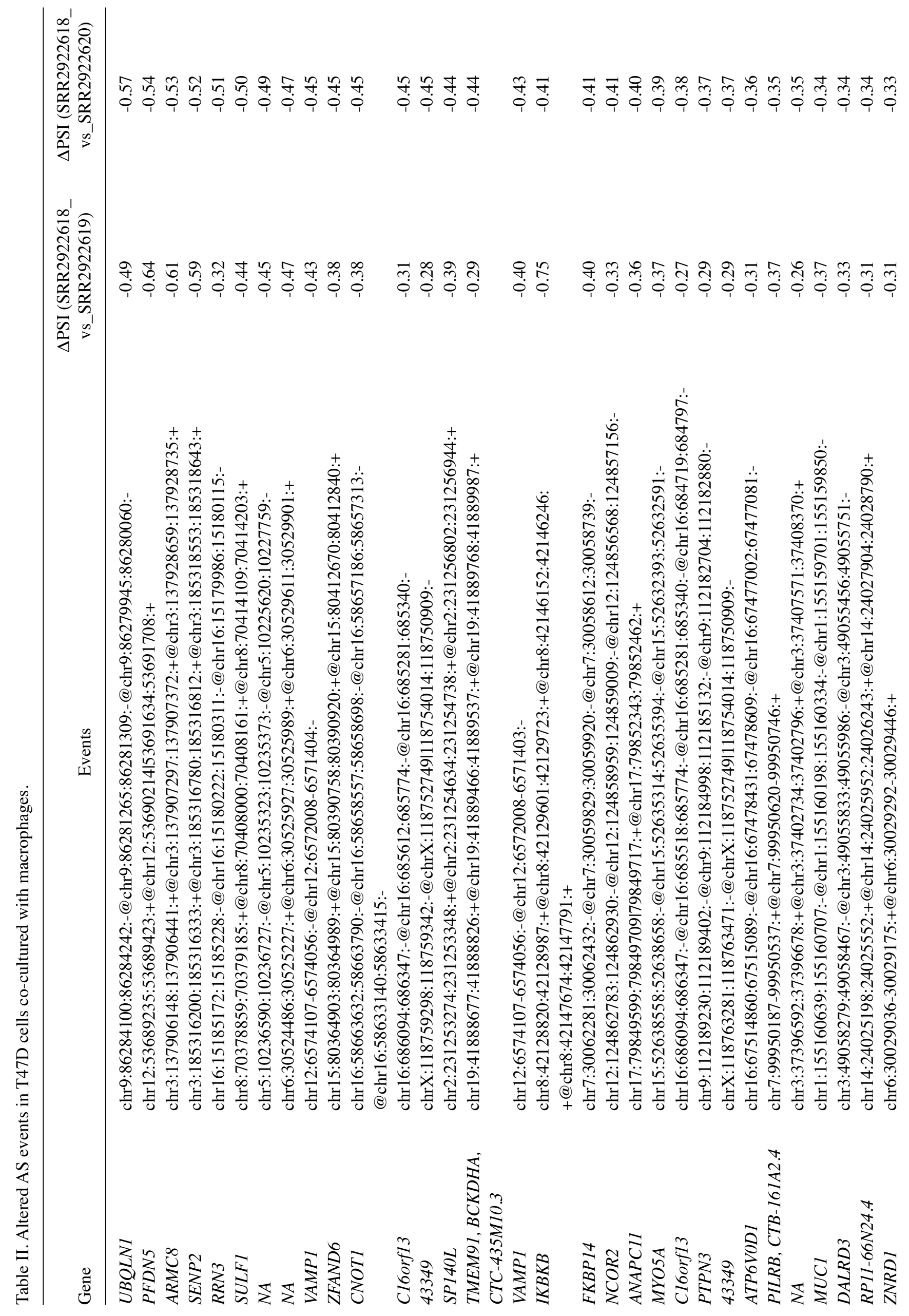




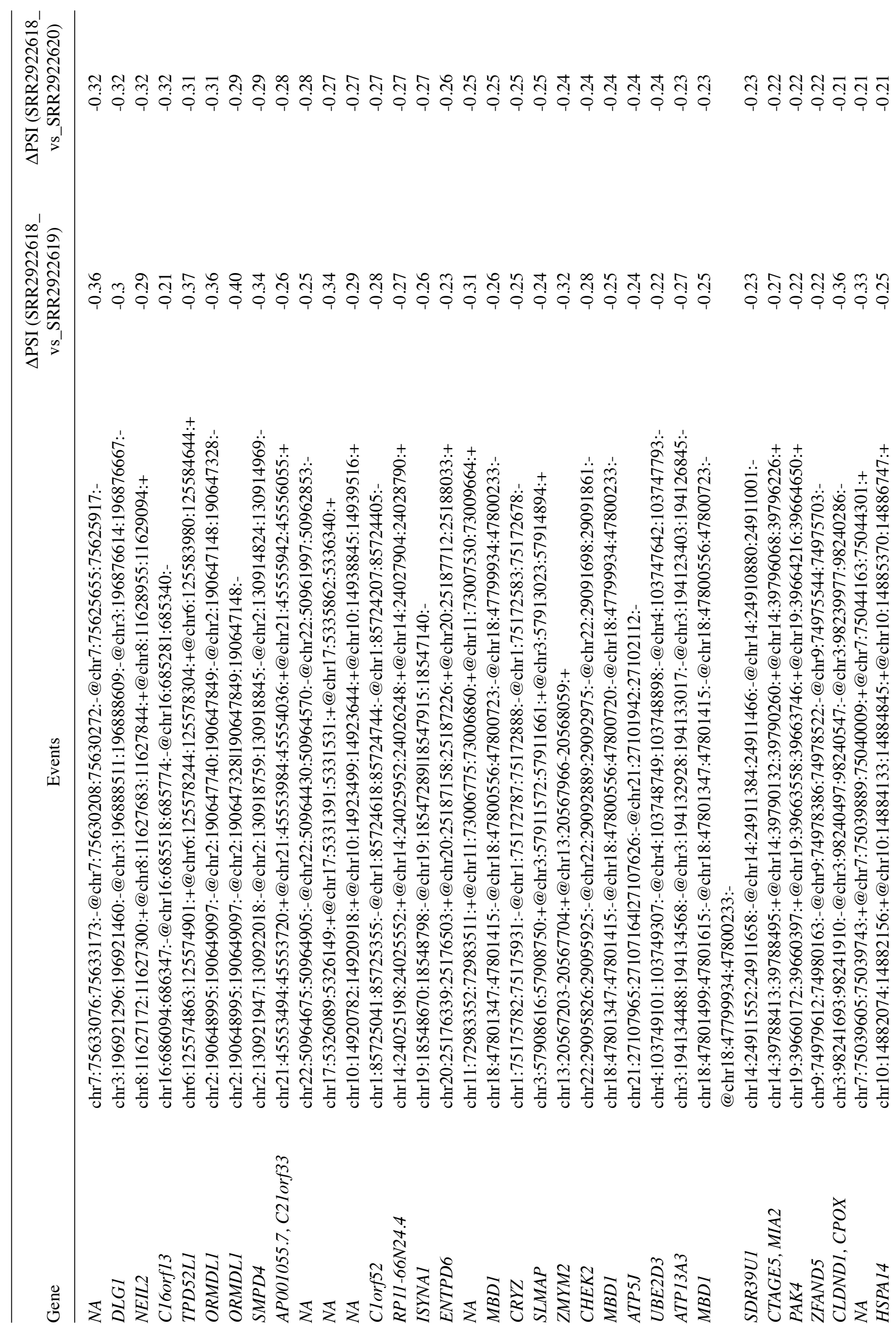




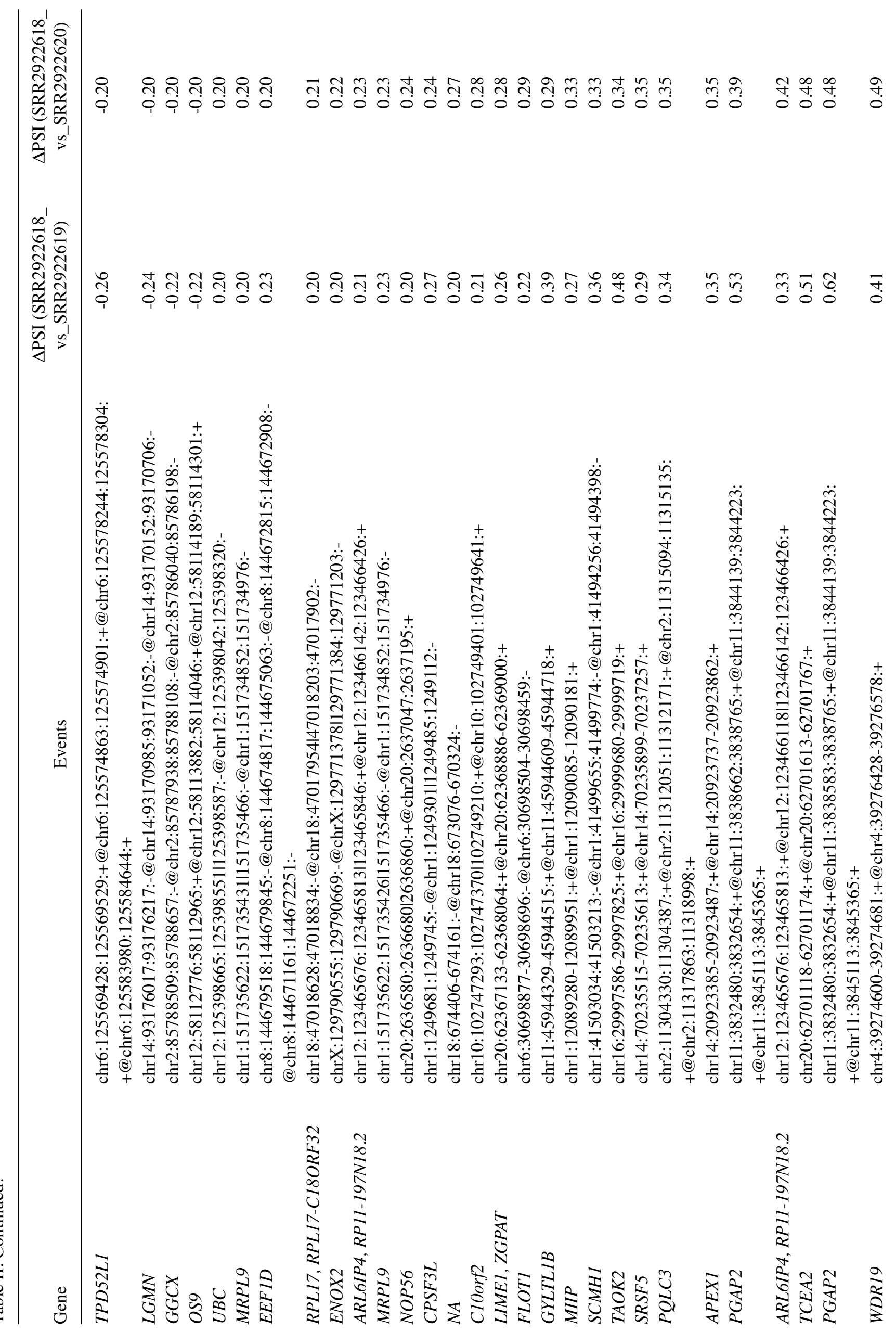


between constitutive skip and altered skip exons and no significant differences among the different treatments (Wilcoxon signed-rank test, P>0.05; Fig. 6C and D). Repetitive elements such as Alu are known to be stochastically exonized (45), and we found Alu elements were more enriched within skip exons in the macrophages cocultured with the T47D cells, were fewer in skip exons of macrophages cocultured with the MDA-MB-231 cells, and were almost absent from skip exons of MDA-MB-231 co-cultured with macrophages and T47D co-cultured with macrophages (Fisher's exact test; Fig. 6E). We conclude that altered skip exons of macrophages cocultured with T47D cells were mostly enriched with Alu element and had highest evolutionary conservation.

Finally, the splicing factors responsible for the AS events in the different cells and treatments were determined. Here, we calculated the associations between splicing factor expression and the PSI values across the 14 samples. The top 5 splicing factors which possessed the most associated splicing events in each of the 4 cell conditions are shown in Fig. 6F. In the MDA-MB-231 cells co-cultured with macrophages, CPSF7, RBM33, SNRNP48, $R B M 43$ and $C S T F 2$ were the top five. Akman et al confirmed that CSTF2 is a major regulator of 3'UTR shortening in TNBC and contributes to the proliferation phenotype (46). In T47D cells co-cultured with macrophages, CWC25, SNRPE, RBM18, $R B M 20$ and $M E X 3 C$ were the top five. Meanwhile, in the macrophages co-cultured with MDA-MB-231 cells, SF3B4, NAA38, SNRPG, ELAVL3 and RBM34 were the top five and in the macrophages co-cultured with T47D cells, $P R P F 3, S F 3 B 2$, SNRNP48,CELF1 and RBM46 were the top five. Lin et al found that knockdown of $C E L F 1$ in primary human macrophages led to increased inflammatory response to M1 stimulation (47). Subsequently, the expression pattern of candidate splicing factors among normal and 4 breast cancer subtypes was explored. Generally, splicing factors responsible for each condition showed similar expression pattern among the samples (Fig. 7A). In addition, we characterized the expression pattern of these splicing factors among samples from the GSE75130 dataset. Splicing factors such as CPSF7, RBM33, SNRNP48 responsible for the altered AS events in MDA-MB-231 cells cocultured with macrophages showed a similar expression pattern among the different treatments (Fig. 7B).

\section{Discussion}

In the present study, mutual editing of alternative splicing (AS) between tumor cells and macrophage was investigated by re-exploring a previous dataset. Importantly, it was found that $\mathrm{ER}^{+}$and triple-negative breast cancer (TNBC) were differentially regulated from the AS events view when co-cultured with macrophages. However, DNA repair and DNA damage processes were enriched in both $\mathrm{ER}^{+}$and $\mathrm{TNBC}$ after co-culturing with macrophages. Meanwhile, macrophages were also differentially regulated from the biological processes view by co-culturing with $\mathrm{ER}^{+}$and TNBC. These results revealed a new view of the mutual regulation between tumor cells and macrophages, in which biological pathways were dramatically different identified at the expression level.

At the expression level, macrophages co-cultured with MDA-MB-231 cells were enriched in the superpathway of citrulline metabolism, cellular effects of sildenafil 


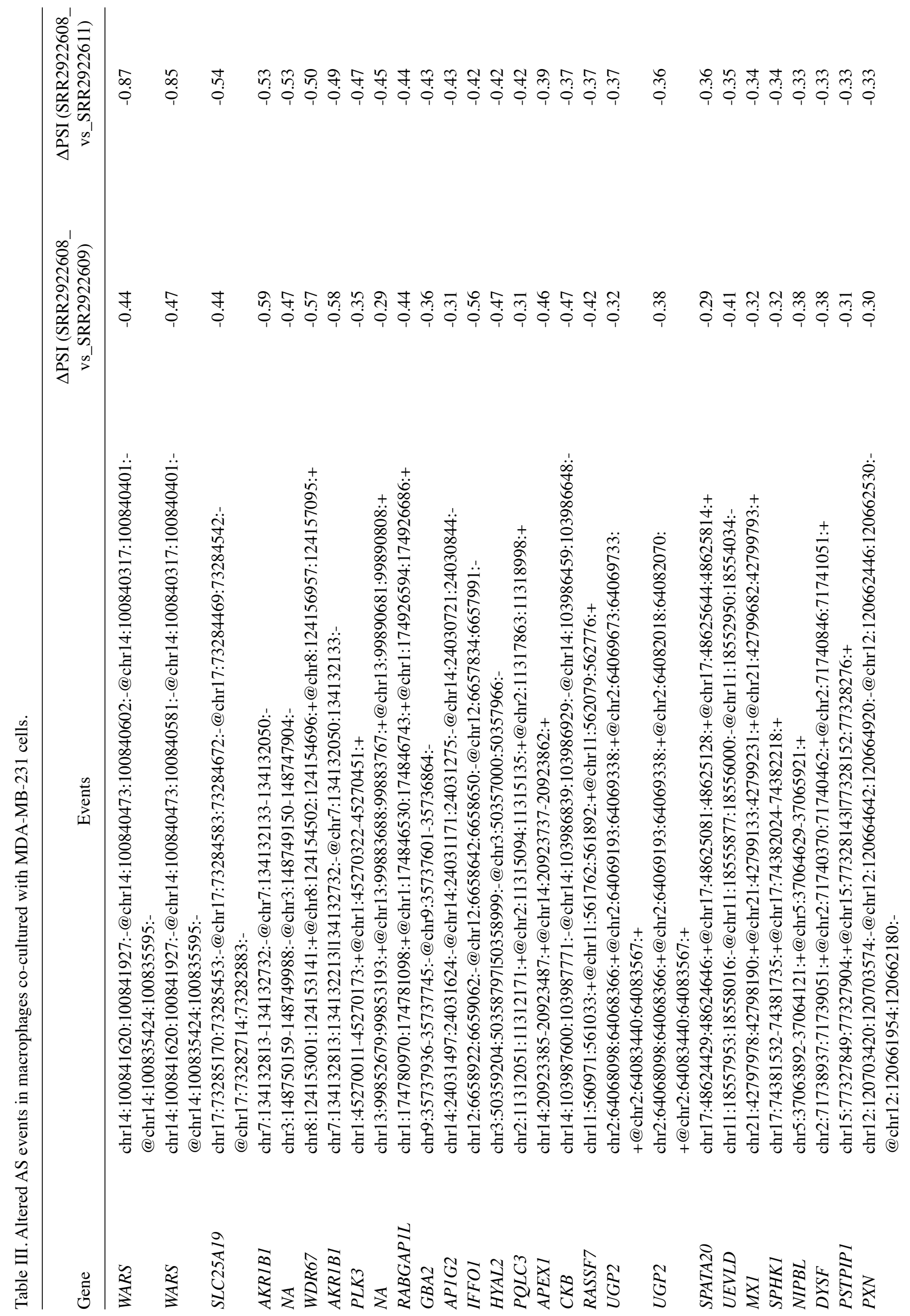




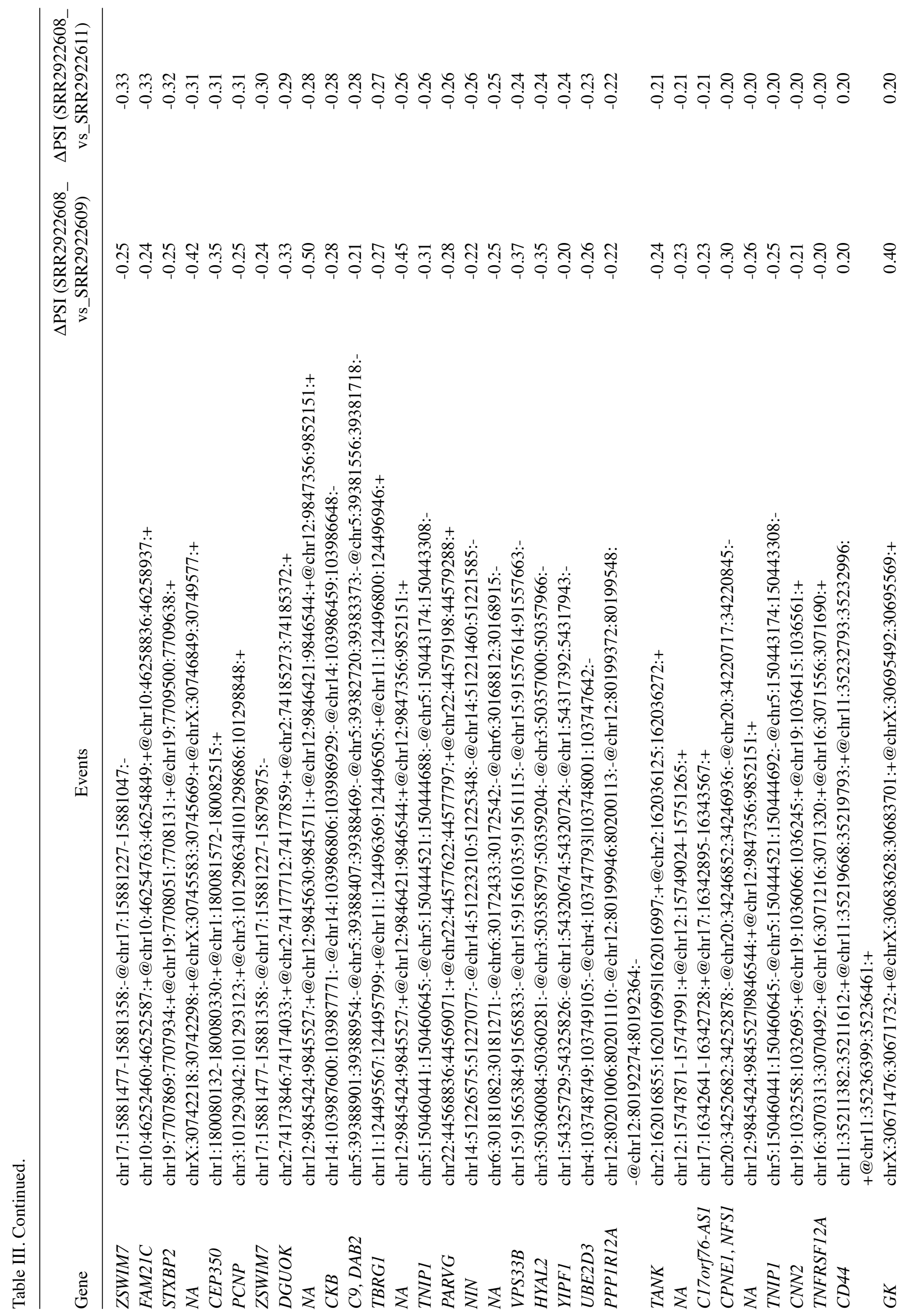




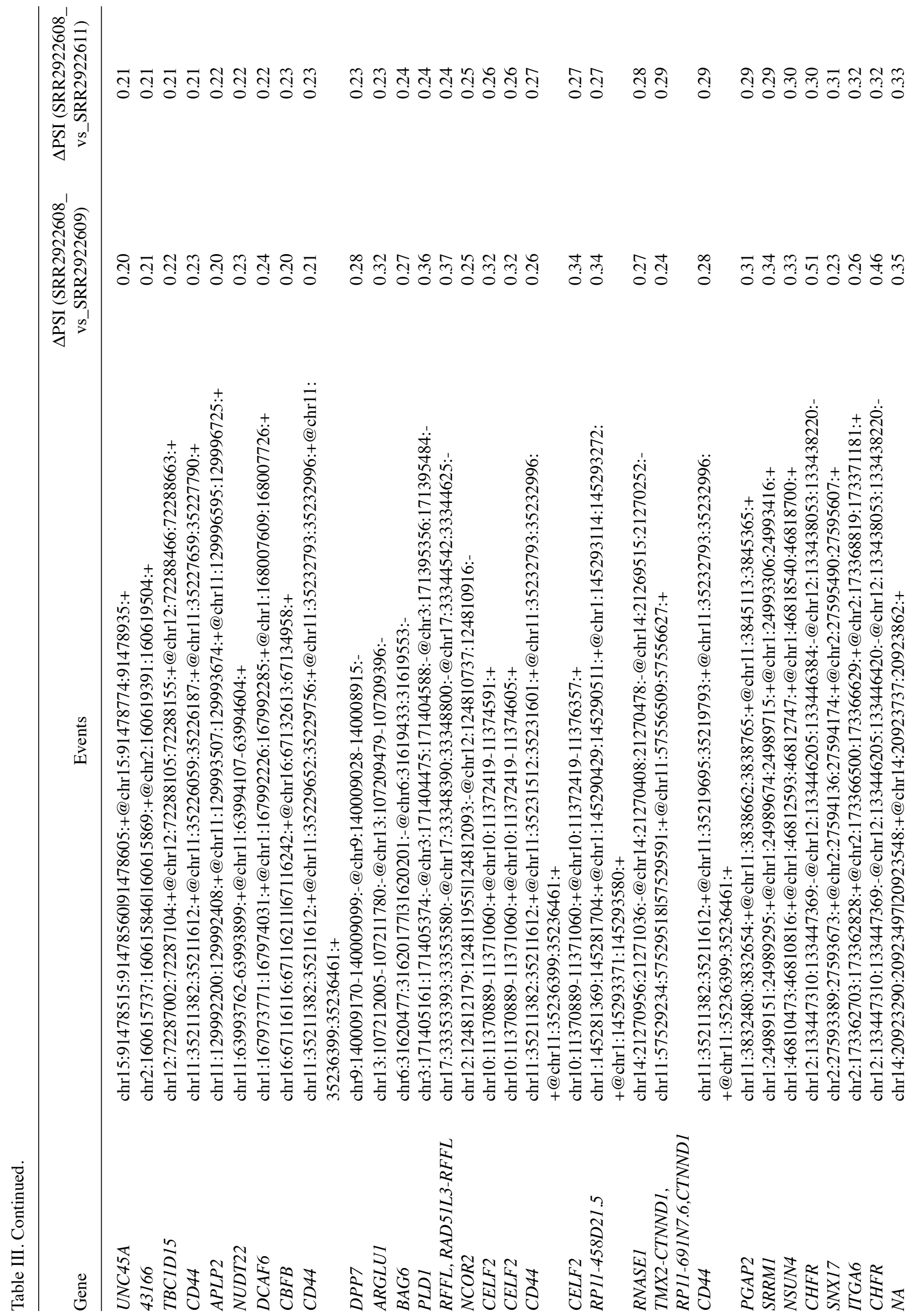




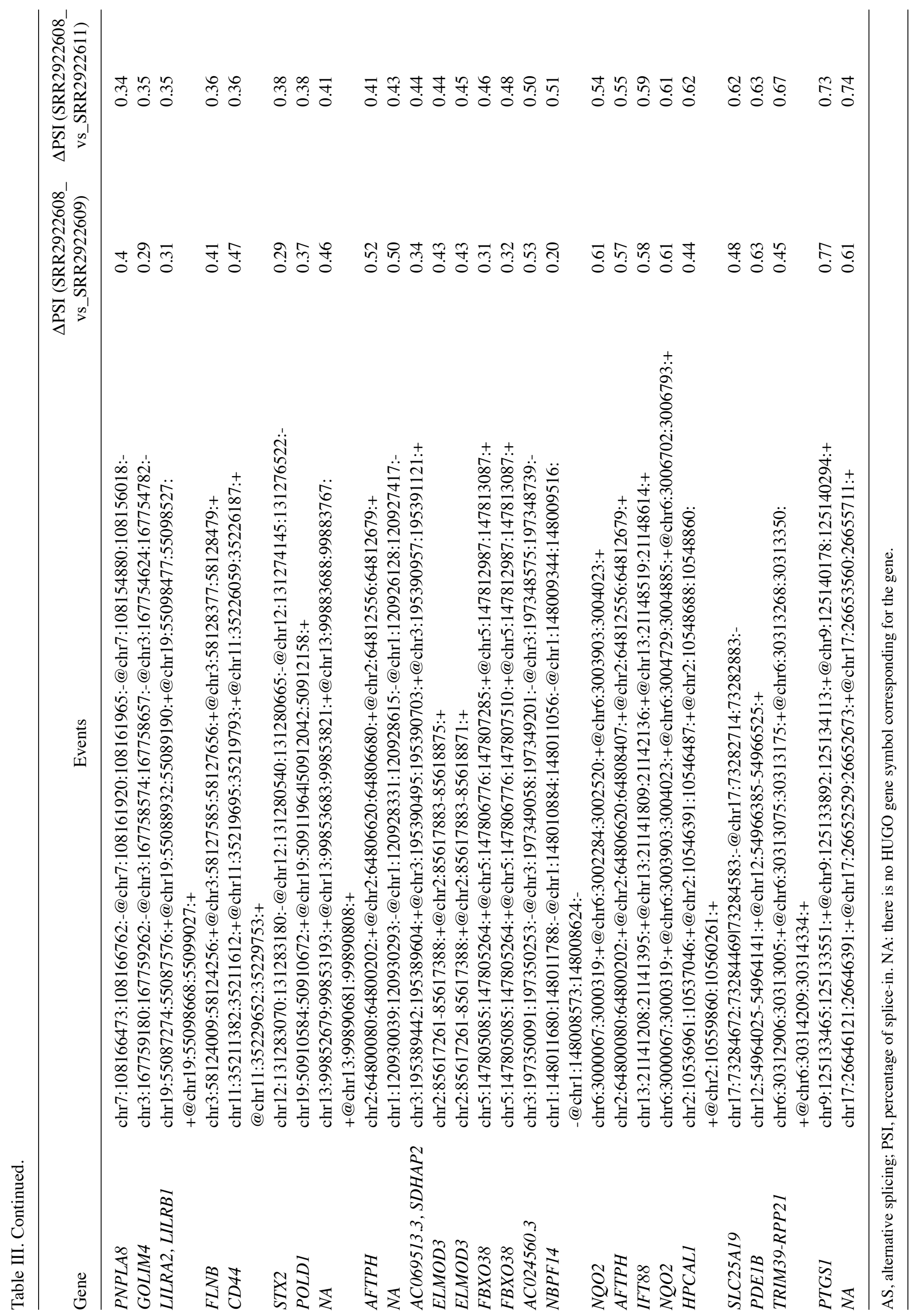




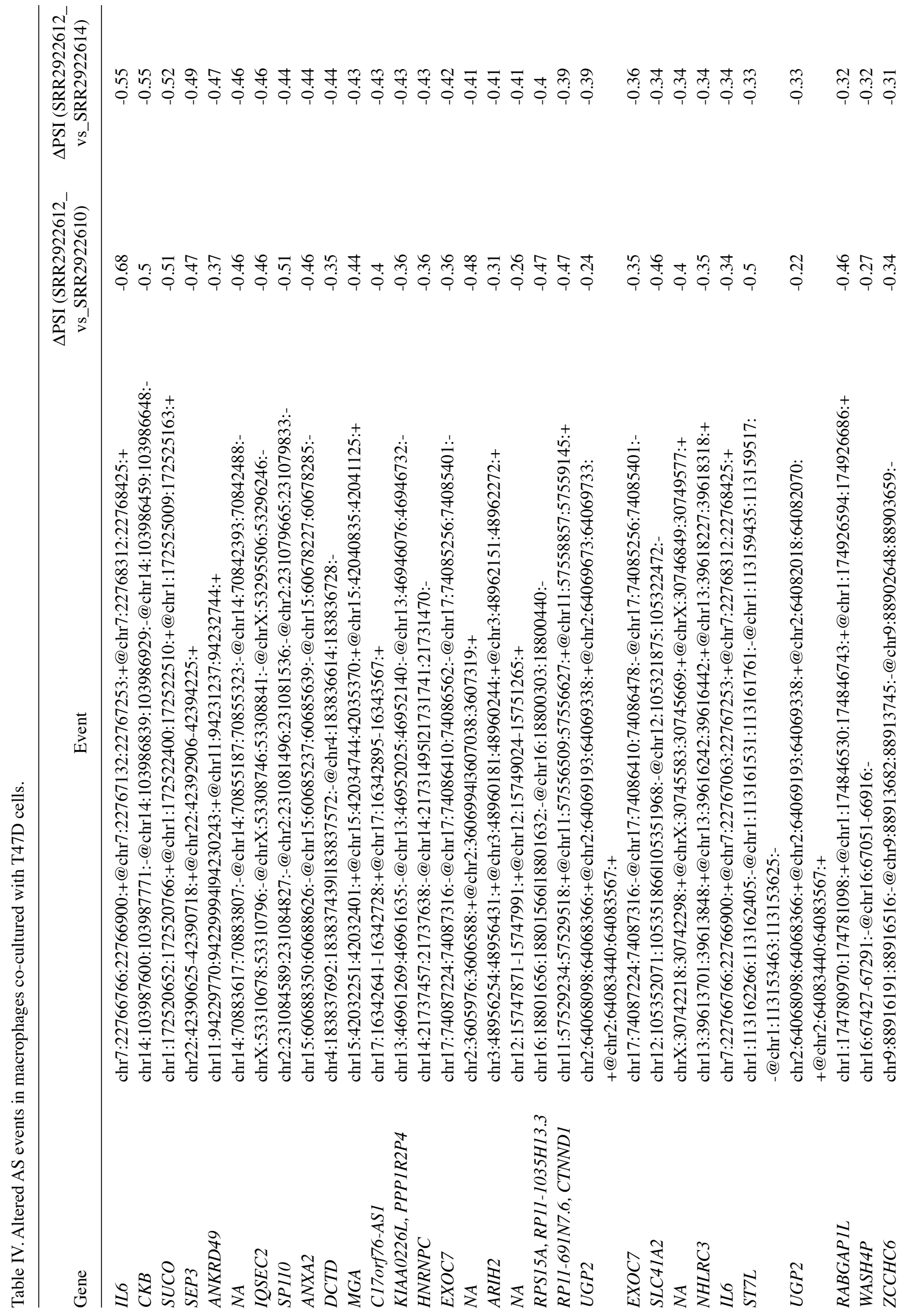




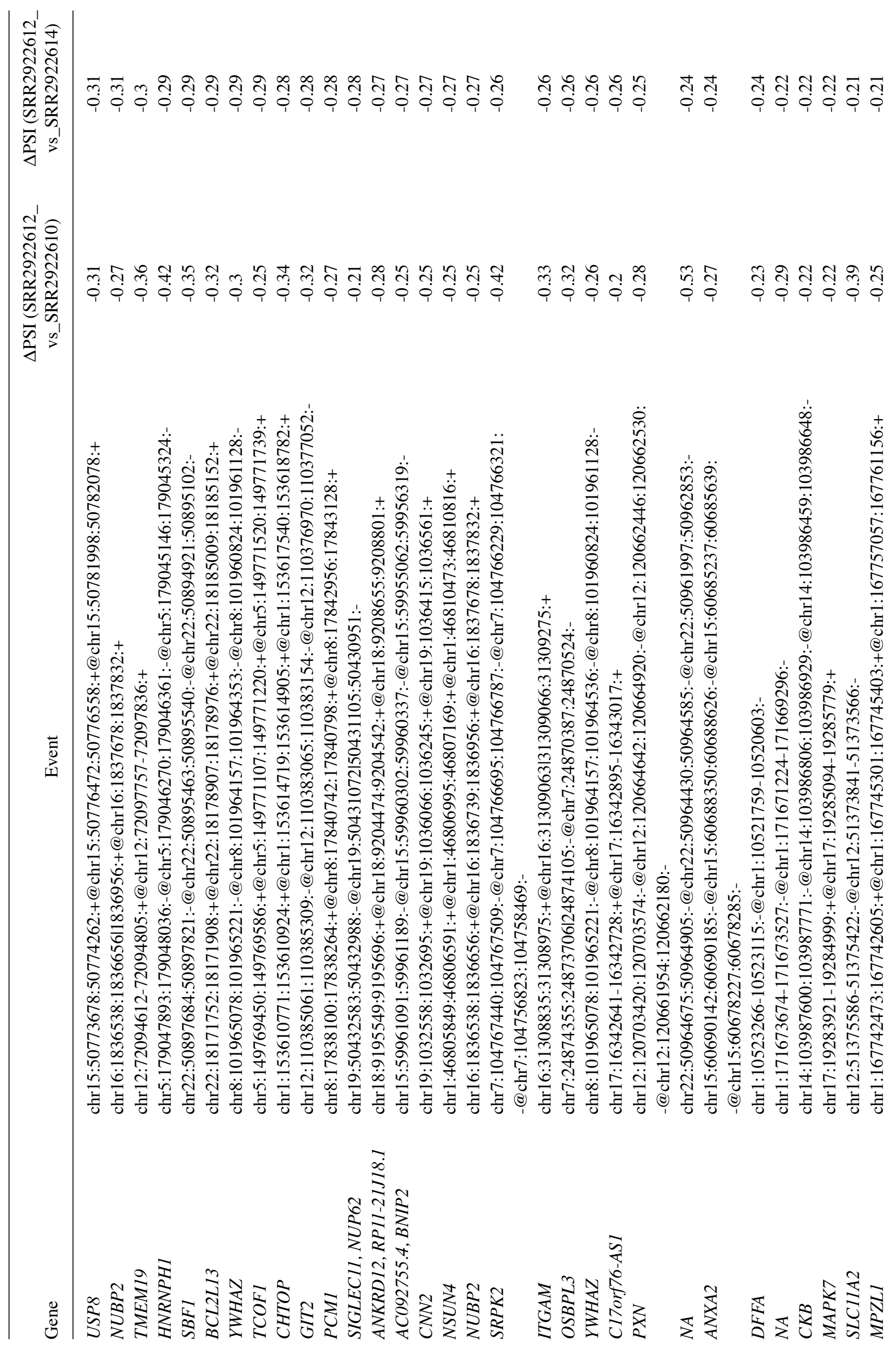




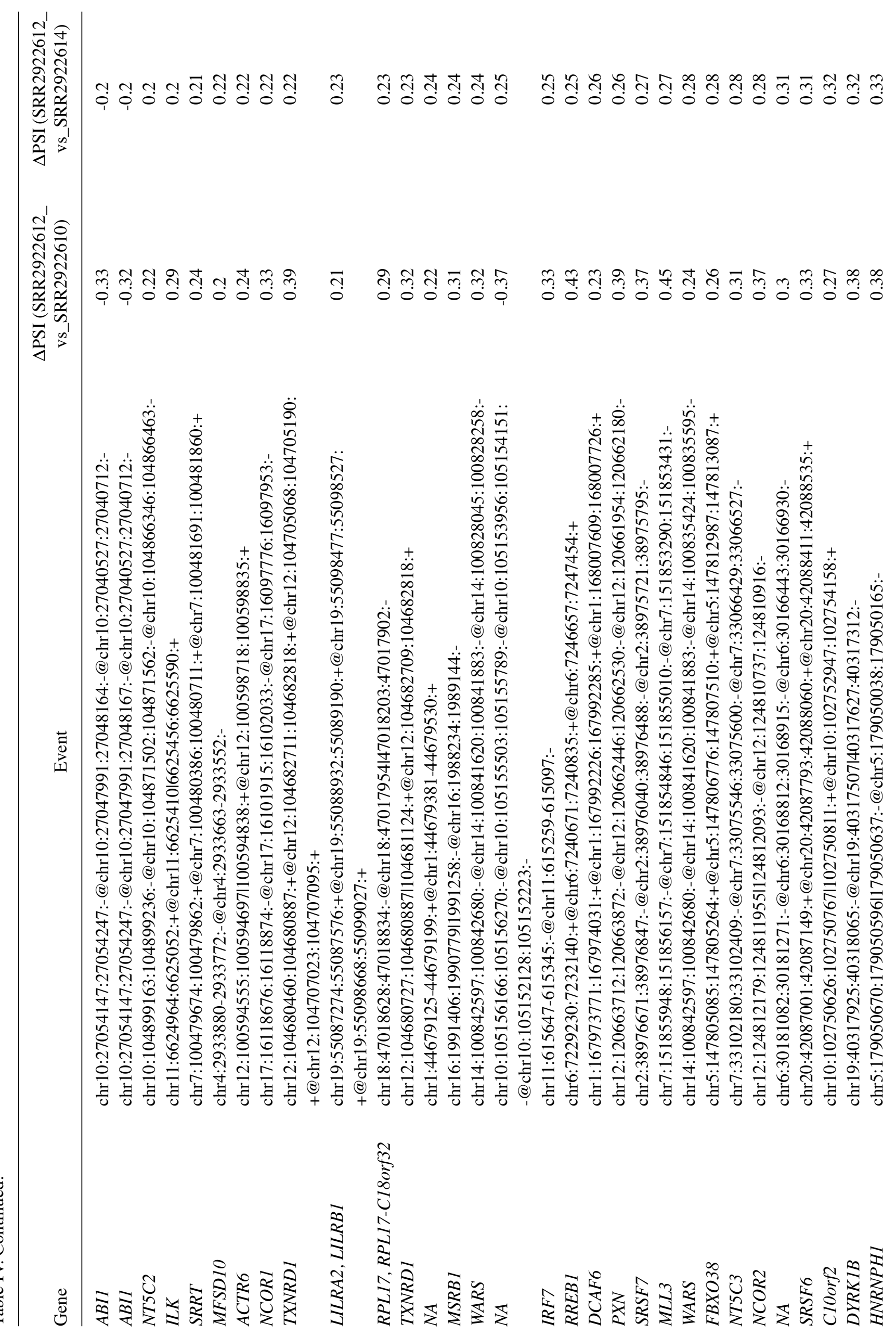




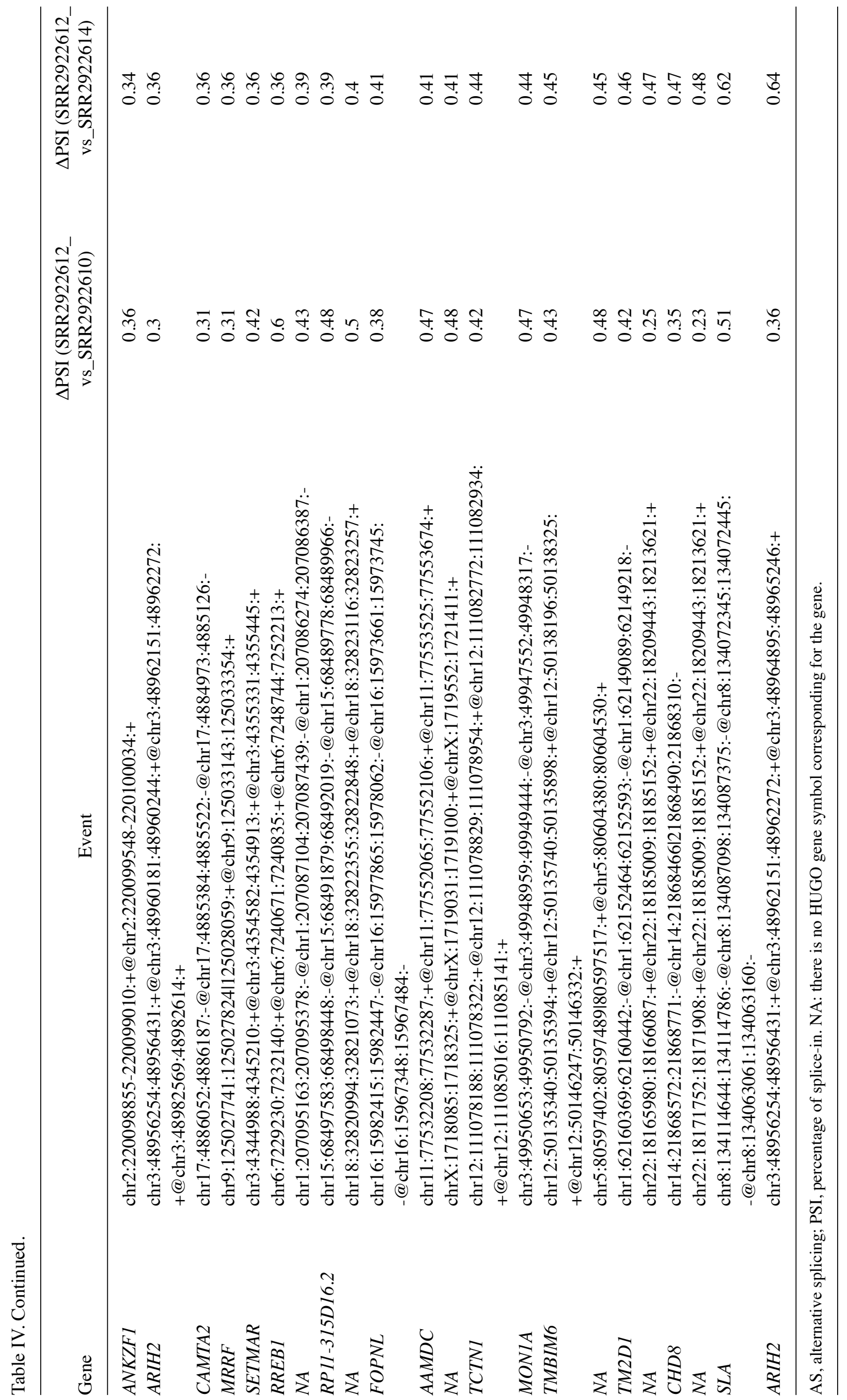



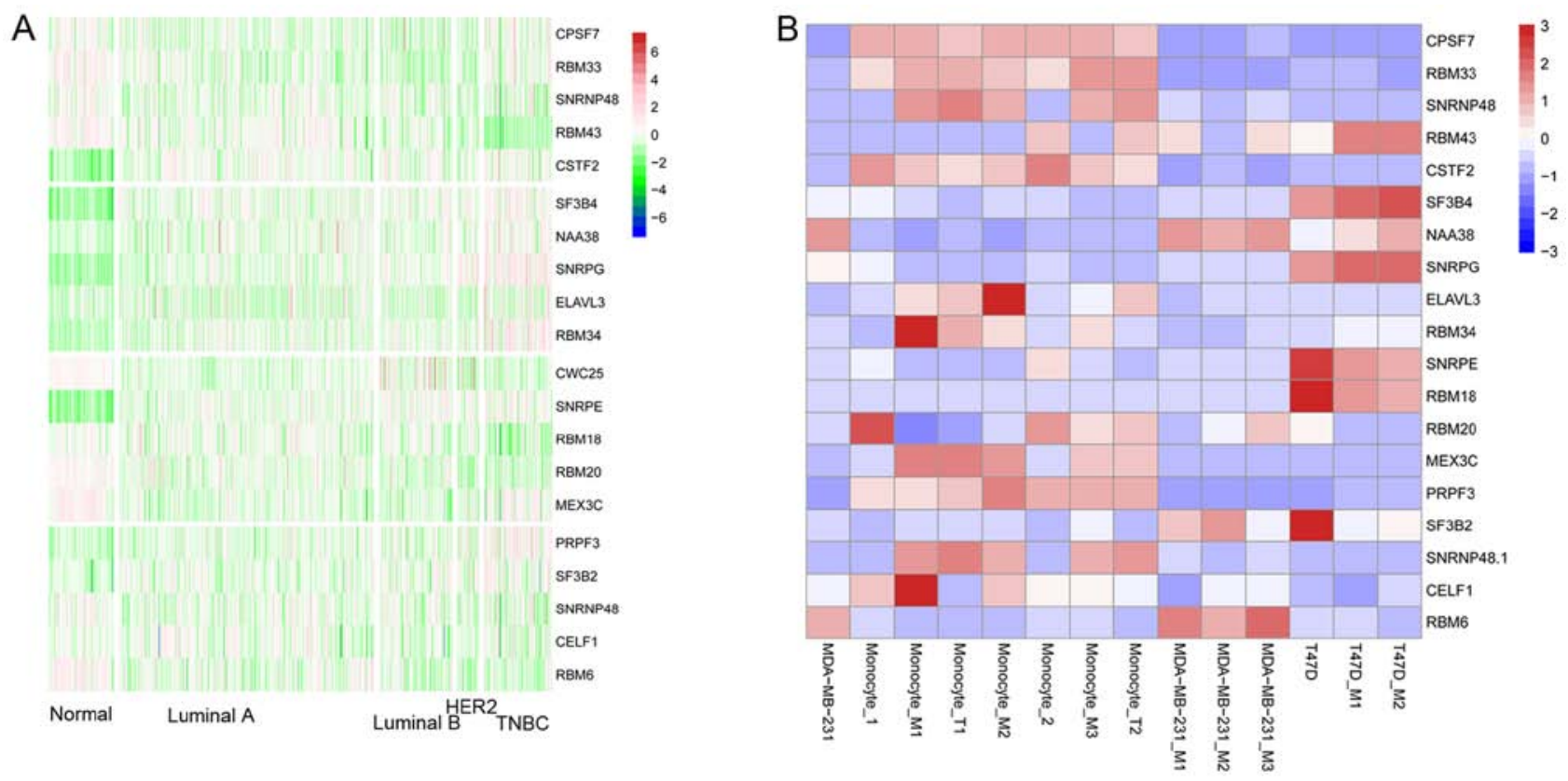

Figure 7. Expression pattern of candidate splicing factors. (A) Expression pattern of candidate splicing factors across normal breast samples and 4 breast cancer subtypes. Expression profiles were derived from BRCA cohort of the TCGA dataset (https://portal.gdc.cancer.gov/). (B) Expression pattern of candidate splicing factors across different treatments from the GSE75130 dataset.

(Viagra), GNRH signaling, citrulline biosynthesis, factors promoting cardiogenesis in vertebrates and bladder cancer signaling. Whereas at the AS level, RNA processing and translation-related processes were enriched. In macrophages co-cultured with T47D cells, biological pathways such as granulocyte adhesion and diapedesis, inhibition of matrix metalloproteases, agranulocyte adhesion and diapedesis, VDR/RXR activation were enriched at the transcription level (21), whereas, biological processes such as protein transport related pathways were enriched at the AS level. In T47D cells co-cultured with macrophages, acute phase response signaling, G-protein coupled receptor signaling, LXR/RXR activation, hepatic fibrosis/hepatic stallate cell activation were enriched at the transcriptional level (21), whereas DNA repair and DNA damage processes were enriched at the AS level. In the MDA-MB-231 cells co-cultured with macrophages, axonal guidance signaling, RhoGDI signaling, relaxin signaling, CCR3 signaling in eosinophil pathways were enriched at the transcriptional level (21), whereas also DNA repair and DNA damage related pathways were enriched at the AS level. Here, we see that the biological pathways affected at the transcription level and alternative splicing level were very different which suggested that AS may provide a new view on the interplay regulation between tumor cells and macrophages.

Another interesting phenomenon was that although the altered AS events from $\mathrm{ER}^{+}$breast cancer and TNBC cells co-cultured with macrophages were not overlapping, the biological pathways such as DNA repair and DNA damage was enriched in both cell line treatments. It has been established that macrophage cells exert their DNA repair functions to normal cells. For example, double-strand breaks (DSBs) activate the cytosolic DNA sensor stimulator of interferon (IFN) genes, thus inducing the production of IFN $\alpha$ and IFN $\beta$, which promote innate immune responses including macrophages (21). In addition, human monocytes, by releasing macrophage-derived heparin-binding epidermal growth factor (HB-EGF), enhance DNA damage response (DDR) in neighboring cells suffering from DNA damage. Consequently, HB-EGF-treated cells exhibit higher double-strand break (DSB) rejoining and display lower levels of residual DSBs (21). This study also has limitations. For example, we did not experimentally verify the functions of candidate splicing factors in regulation of AS profiles under different treatments. In addition, the roles of key AS events in the cross-talk between breast cancer cells and macrophages warrant further research. Further investigation of the functions of splicing factors and key altered AS events would largely improve our understanding of the molecular mechanisms involved in cancer-macrophage crosstalk.

In short, our results here showed that macrophages play roles in the regulation of DNA damage and DNA repair pathways, which improve our understanding concerning the interplay between macrophages and tumor cells. Thus, the specific roles of macrophages in the regulation of tumor cells require further research, which may shed light on the improvement of cancer immunotherapy.

\section{Acknowledgements}

Not applicable.

\section{Funding}

The present study was funded by the Yunnan Applied Basic Research Projects (grant no. 2016FB038) and the National Natural Science Foundation of China (grant no. 31801249).

\section{Availability of data and materials}

The datasets generated during this study are available from the corresponding author upon reasonable request. 


\section{Authors' contributions}

LD and $\mathrm{HZ}$ designed the experiments, interpreted the results, and wrote the manuscript. WD and DL performed the data analysis. PZ, LS, HD, YL, XB and YW revised this work critically and helped to interpret the data. All authors read and approved the manuscript and agree to be accountable for all aspects of the research in ensuring that the accuracy or integrity of any part of the work are appropriately investigated and resolved.

\section{Ethics approval and consent to participate}

Not applicable.

\section{Patient consent for publication}

Not applicable.

\section{Competing interests}

All the authors declare that there was no financial support or relationship that may pose a conflict of interest and all the authors have no conflict of interests.

\section{References}

1. Spill F, Reynolds DS, Kamm RD and Zaman MH: Impact of the physical microenvironment on tumor progression and metastasis. Curr Opin Biotechnol 40: 41-48, 2016.

2. Qian BZ and Pollard JW: Macrophage diversity enhances tumor progression and metastasis. Cell 141: 39-51, 2010.

3. Danhier F, Feron O and Preat V: To exploit the tumor microenvironment: Passive and active tumor targeting of nanocarriers for anti-cancer drug delivery. J Control Release 148: 135-146, 2010.

4. Engblom C, Pfirschke C and Pittet MJ: The role of myeloid cells in cancer therapies. Nat Rev Cancer 16: 447-462, 2016.

5. Mills CD: M1 and M2 macrophages: Oracles of health and disease. Crit Rev Immunol 32: 463-488, 2012.

6. Galdiero MR, Bonavita E, Barajon I, Garlanda C, Mantovani A and Jaillon S: Tumor associated macrophages and neutrophils in cancer. Immunobiology 218: 1402-1410, 2013.

7. Balkwill F, Charles KA and Mantovani A: Smoldering and polarized inflammation in the initiation and promotion of malignant disease. Cancer Cell 7: 211-217, 2005.

8. Mantovani A, Allavena P, Sica A and Balkwill F: Cancer-related inflammation. Nature 454: 436-444, 2008.

9. Zhang W, Wang LA, Zhou DB, Cui QC, Zhao DC and Wu YJ: Expression of tumor-associated macrophages and vascular endothelial growth factor correlates with poor prognosis of peripheral T-cell lymphoma, not otherwise specified. Leukemia Lymphoma 52: 46-52, 2011.

10. Zhang BC, Gao J, Wang J, Rao ZG, Wang BC and Gao JF: Tumor-associated macrophages infiltration is associated with peritumoral lymphangiogenesis and poor prognosis in lung adenocarcinoma. Med Oncol 28: 1447-1452, 2011.

11. Azizi E, Carr AJ, Plitas G, Cornish AE, Konopacki C, Prabhakaran S, Nainys J, Wu K, Kiseliovas V, Setty M, et al: Single-cell map of diverse immune phenotypes in the breast tumor microenvironment. Cell 174: 1293-1308.e36, 2018.

12. Lin EY, Li JF, Gnatovskiy L, Deng Y, Zhu L, Grzesik DA, Qian H, Xue XN and Pollard JW: Macrophages regulate the angiogenic switch in a mouse model of breast cancer. Cancer Res 66: 11238-11246, 2006.

13. Lin EY, Nguyen AV, Russell RG and Pollard JW: Colonystimulating factor 1 promotes progression of mammary tumors to malignancy. J Exp Med 193: 727-740, 2001.

14. Qian BZ, Li J, Zhang H, Kitamura T, Zhang J, Campion LR, Kaiser EA, Snyder LA and Pollard JW: CCL2 recruits inflammatory monocytes to facilitate breast-tumour metastasis. Nature 475 : 222-225, 2011
15. Cortez-Retamozo V, Etzrodt M, Newton A, Ryan R, Pucci F, Sio SW, Kuswanto W, Rauch PJ, Chudnovskiy A, Iwamoto Y, et al: Angiotensin II drives the production of tumor-promoting macrophages. Immunity 38: 296-308, 2013.

16. Oltean S and Bates DO: Hallmarks of alternative splicing in cancer. Oncogene 33: 5311-5318, 2014.

17. Liu HF, Lorenzini PA, Zhang F, Xu S, Wong MS, Zheng J and Roca X: Alternative splicing analysis in human monocytes and macrophages reveals MBNL1 as major regulator. Nucleic Acids Res 46: 6069-6086, 2018.

18. Shadle PJ, Aldwin L, Nitecki DE and Koths K: Human macrophage colony-stimulating factor heterogeneity results from alternative mRNA splicing, differential glycosylation, and proteolytic processing. J Cell Biochem 40: 91-107, 1989.

19. Leek RD, Lewis CE, Whitehouse R, Greenall M, Clarke J and Harris AL: Association of macrophage infiltration with angiogenesis and prognosis in invasive breast carcinoma. Cancer Res 56: 4625-4629, 1996.

20. Mahmoud SM, Lee AH, Paish EC, Macmillan RD, Ellis IO and Green AR: Tumour-infiltrating macrophages and clinical outcome in breast cancer. J Clin Pathol 65: 159-163, 2012.

21. Hollmen M, Roudnicky F, Karaman S and Detmar M: Characterization of macrophage-cancer cell crosstalk in estrogen receptor positive and triple-negative breast cancer. Sci Rep 5: 9188, 2015

22. Galperin MY, Fernandez-Suarez XM and Rigden DJ: The 24th annual Nucleic Acids Research database issue: A look back and upcoming changes. Nucleic Acids Res 45: 5627-5627, 2017.

23. Dobin A, Davis CA, Schlesinger F, Drenkow J, Zaleski C, Jha S, Batut P, Chaisson M and Gingeras TR: STAR: Ultrafast universal RNA-seq aligner. Bioinformatics 29: 15-21, 2013.

24. Liao Y, Smyth GK and Shi W: FeatureCounts: An efficient general purpose program for assigning sequence reads to genomic features. Bioinformatics 30: 923-930, 2014.

25. Robinson MD, McCarthy DJ and Smyth GK: edgeR: A bioconductor package for differential expression analysis of digital gene expression data. Bioinformatics 26: 139-140, 2010.

26. Katz Y, Wang ET, Airoldi EM and Burge CB: Analysis and design of RNA sequencing experiments for identifying isoform regulation. Nat Methods 7: 1009-1015, 2010.

27. Lever J, Krzywinski M and Atman N: Points of Significance principal component analysis. Nat Methods 14: 641-642, 2017.

28. Hotelling $\mathrm{H}$ : Analysis of a complex of statistical variables into principal components. J Educ Psychol 24: 417-441, 1933.

29. Ginestet C: ggplot2: Elegant graphics for data analysis. J R Stat Soc A Stat 174: 245-245, 2011.

30. Huang DW, Sherman BT and Lempicki RA: Systematic and integrative analysis of large gene lists using DAVID bioinformatics resources. Nat Protoc 4: 44-57, 2009

31. Szklarczyk D, Morris JH, Cook H, Kuhn M, Wyder S, Simonovic M, Santos A, Doncheva NT, Roth A, Bork P, et al: The STRING database in 2017: Quality-controlled protein-protein association networks, made broadly accessible. Nucleic Acids Res 45: D362-D368, 2017.

32. Marchler-Bauer A,Bo Y,Han L,He J,LanczyckiCJ,Lu S,ChitsazF, Derbyshire MK, Geer RC, Gonzales NR, et al: CDD/SPARCLE: Functional classification of proteins via subfamily domain architectures. Nucleic Acids Res 45: D200-D203, 2017.

33. Kent WJ, Zweig AS, Barber G, Hinrichs AS and Karolchik D: BigWig and BigBed: Enabling browsing of large distributed datasets. Bioinformatics 26: 2204-2207, 2010.

34. Quinlan AR and Hall IM: BEDTools: A flexible suite of utilities for comparing genomic features. Bioinformatics 26: 841-842, 2010.

35. Eng L, Coutinho G, Nahas S, Yeo G, Tanouye R, Babaei M, Dörk T, Burge C and Gatti RA: Nonclassical splicing mutations in the coding and noncoding regions of the ATM gene: Maximum entropy estimates of splice junction strengths. Hum Mutat 23: 67-76, 2004.

36. Domazet-Loso T and Tautz D: An ancient evolutionary origin of genes associated with human genetic diseases. Mol Biol Evol 25 2699-2707, 2008.

37. Cai Z, Chehab NH and Pavletich NP: Structure and activation mechanism of the CHK2 DNA damage checkpoint kinase. Mol Cell 35: 818-829, 2009.

38. Nevanlinna $\mathrm{H}$ and Bartek J: The CHEK2 gene and inherited breast cancer susceptibility. Oncogene 25: 5912-5919, 2006.

39. Zhao H, Traganos F and Darzynkiewicz Z: Phosphorylation of p53 on Ser15 during cell cycle caused by Topo I and Topo II inhibitors in relation to ATM and Chk2 activation. Cell Cycle 7: 3048-3055, 2008. 
40. Meijers-Heijboer H, van den Ouweland A, Klijn J, Wasielewski M, de Snoo A, Oldenburg R, Hollestelle A, Houben M, Crepin E, van Veghel-Plandsoen M, et al: Low-penetrance susceptibility to breast cancer due to CHEK2 $(*) 1100 \mathrm{delC}$ in noncarriers of BRCA1 or BRCA2 mutations. Nat Genet 31: 55-59, 2002.

41. Pevsner J, Hsu SC, Hyde PS and Scheller RH: Mammalian homologues of yeast vacuolar protein sorting (vps) genes implicated in Golgi-to-lysosome trafficking. Gene 183: 7-14, 1996.

42. Halachmi N and Lev Z: The Secl family: A novel family of proteins involved in synaptic transmission and general secretion. J Neurochem 66: 889-897, 1996.

43. Akbar MA, Mandraju R, Tracy C, Hu W, Pasare C and Krämer H: ARC Syndrome-linked Vps33B protein is required for inflammatory endosomal maturation and signal termination. Immunity 45 : 267-279, 2016

44. Boisson B, Honda Y, Ajiro M, Bustamante J, Bendavid M Gennery AR, Kawasaki Y, Ichishima J, Osawa M, Nihira H, et al: Rescue of recurrent deep intronic mutation underlying cell type-dependent quantitative NEMO deficiency. J Clin Invest 129: 583-597, 2019
45. Stower H: Alternative splicing: Regulating Alu element 'exonization' Nature Rev Genet 14: 152-153, 2013.

46. Akman HB, Öyken M, Tuncer T, Can T and Erson-Bensan AE: 3'UTR shortening and EGF signaling: Implications for breast cancer. Hum Mol Genet 24: 6910-6920, 2015.

47. Lin J, Hu Y, Nunez S, Foulkes AS, Cieply B, Xue C, Gerelus M, Li W, Zhang H, Rader DJ, et al: Transcriptome-wide analysis reveals modulation of human macrophage inflammatory phenotype through alternative splicing. Arterioscl Throm Vas Biol 36: 1434-1347, 2016

(i) (5) $\odot$ This work is licensed under a Creative Commons Attribution-NonCommercial-NoDerivatives 4.0 International (CC BY-NC-ND 4.0) License. 